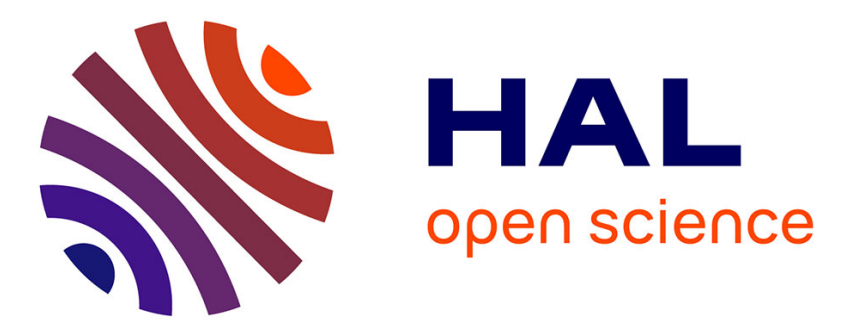

\title{
Fluorescent nanodiamond tracking reveals intraneuronal transport abnormalities induced by brain-disease-related genetic risk factors
}

\author{
Simon Haziza, Nitin Mohan, Yann Loe-Mie, Aude-Marie Lepagnol-Bestel, \\ Sophie Massou, Marie-Pierre Adam, Xuan Loc Le, Julia Viard, Christine \\ Plancon, Rachel Daudin, et al.
}

\section{To cite this version:}

Simon Haziza, Nitin Mohan, Yann Loe-Mie, Aude-Marie Lepagnol-Bestel, Sophie Massou, et al.. Fluorescent nanodiamond tracking reveals intraneuronal transport abnormalities induced by brain-disease-related genetic risk factors. Nature Nanotechnology, 2017, 12 (4), pp.322-328. 10.1038/nnano.2016.260 . pasteur-02873510

\section{HAL Id: pasteur-02873510}

https://hal-pasteur.archives-ouvertes.fr/pasteur-02873510

Submitted on 22 Dec 2020

HAL is a multi-disciplinary open access archive for the deposit and dissemination of scientific research documents, whether they are published or not. The documents may come from teaching and research institutions in France or abroad, or from public or private research centers.
L'archive ouverte pluridisciplinaire HAL, est destinée au dépôt et à la diffusion de documents scientifiques de niveau recherche, publiés ou non, émanant des établissements d'enseignement et de recherche français ou étrangers, des laboratoires publics ou privés.

\section{(ㄷ)(1) $\$$}

Distributed under a Creative Commons Attribution - NonCommerciall 4.0 International 


\section{Fluorescent nanodiamond tracking reveals intraneuronal transport abnormalities induced by brain disease-related genetic risk factors}

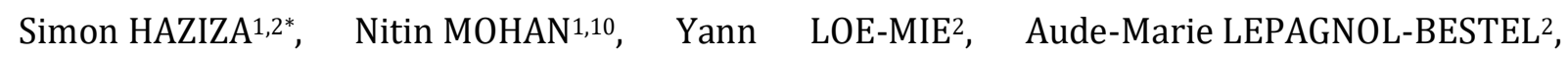
Sophie MASSOU1,11, Marie-Pierre ADAM1,12, Xuan Loc LE1 ${ }^{1}$, Julia VIARD², Christine PLANCON³, Rachel DAUDIN ${ }^{2}$, Pascale KOEBEL ${ }^{4}$, Emilie DORARD ${ }^{2}$, Christiane ROSE², Feng-Jen HSIEH ${ }^{5}$, ChihChe WU6, Brigitte POTIER ${ }^{2}$, Yann HERAULT ${ }^{4}$, Carlo SALA7, Aiden CORVIN, Bernadette ALLINQUANT $^{2}$, Huan-Cheng CHANG $^{5}$, François TREUSSART ${ }^{1 *}$ and Michel SIMONNEAU ${ }^{1,2,9^{*}+}$

${ }^{1}$ Laboratoire Aimé Cotton, CNRS, Univ. Paris-Sud, ENS Cachan, Université Paris-Saclay, 91405 Orsay, France

2 Centre de Psychiatrie et Neurosciences, INSERM U894, Université Paris-Descartes, 75014 Paris, France

${ }^{3}$ Centre National de Génotypage, 91057 Evry, France

${ }^{4}$ Institut de génétique et de biologie moléculaire et cellulaire, CNRS UMR 7104, INSERM U 964, Université de Strasbourg, 67400 Illkirch-Graffenstaden, France

${ }^{5}$ Institute of Atomic and Molecular Sciences, Academia Sinica, Taipei 106, Taiwan

${ }^{6}$ Department of Applied Chemistry, National Chi Nan University, Puli, Nantou Hsien 545, Taiwan

${ }^{7}$ Neuroscience Institute, CNR, 20129 Milano, Italy

8 Department of Psychiatry, Institute of Neuroscience, Trinity College Dublin, Dublin 2, Ireland

${ }^{9}$ Department of Biology, ENS Cachan, Université Paris-Saclay, 94235 Cachan, France

${ }^{10}$ Current address: Institut de Ciències Fotòniques (ICFO), 08860 Castelledefels, Spain

11 Current address: Institut Interdisciplinaire de Neurosciences, CNRS, Université de Bordeaux, 33077 Bordeaux, France

12 Current address: European Laboratory for Non-Linear Spectroscopy, University of Florence, 50019 Sesto Fiorentino, Italy

†These authors contributed equally to this work.

*emails: michel.simonneau@inserm.fr (M.S.), francois.treussart@ens-cachan.fr (F.T.) and simon.haziza@ens-cachan.fr (S.H.) 
Brain diseases such as autism and Alzheimer's disease (each inflicting $>1 \%$ of the world population) involves a large network of genes displaying subtle changes in their expression ${ }^{1}$ Abnormalities in intraneuronal transport have been linked to genetic risk factors found in patients ${ }^{2,3}$, suggesting the relevance of measuring this key biological process. However, current techniques are not sensitive enough to detect minor abnormalities. Here, we report a sensitive method to measure changes in intraneuronal transport induced by brain disease-related genetic risk factors using fluorescent nanodiamonds (fNDs). We show that the high brightness, photostability and absence of cytotoxicity ${ }^{4}$ allow fNDs to be tracked inside the branches of dissociated neurons with $12 \mathrm{~nm}$ spatial and $50 \mathrm{~ms}$ time resolutions. As proof-of-principle, we applied the fND-tracking assay on two transgenic mouse lines that mimic the slight changes in protein concentration $(\sim 30 \%)$ found in brains of patients. In both cases, we show that the fND assay is sufficiently sensitive to detect these changes.

A polygenic architecture of brain diseases as diverse as autism spectrum disorders, schizophrenia and late-onset Alzheimer's disease is emerging, with the discovery of interplay between hundreds of genetic variants and subtle changes in gene expression, later referred as genetic risk factors ${ }^{1}$. The modelling of human brain diseases to understand the function of related genes usually relies on the establishment of transgenic mouse mimicking the effects of genetic changes ${ }^{5}$. As intraneuronal transport abnormalities have long been recognized to be linked to neuropsychiatric and neurodegenerative diseases ${ }^{2,3}$, we decided to mimic in vivo the changes induced by brain disease-related genetic variants and subsequently quantify their impact on the intraneuronal transport using primary cultures of mouse hippocampal neurons.

Previous attempts to quantify intraneuronal transport have been based on cargo labelling with genetic constructs of chimeric fluorescent proteins ${ }^{6}$. Limitations of this strategy are low transfection yield, uncontrolled protein overproduction, photobleaching of the reporters, and substantial cytotoxicity. Fluorescent semiconductor nanocrystals (quantum dots, QDs) have been widely used to probe a variety of cellular events ${ }^{7}$, owing to their high photoluminescence yield and good photostability. However, attempts to use QDs to study intraneuronal transport have been limited to the specific model of long axons of dorsal root neurons cultured in microfluidic devices ${ }^{8}$. Moreover, QDs blinking impedes high spatiotemporal resolution tracking and therefore biases transport parameter 
measurements as we show in this letter. Considering these intrinsic limitations, there is a need for developing new generic approaches able to screen the functional impact of brain disease-related genetic modifications.

Here, we developed a quantitative assay based on tracking of fluorescent diamond nanocrystals (fluorescent NanoDiamond, fND) in mouse hippocampal neurons (Fig. 1, Supplementary Videos 1 and 2). We used $30 \mathrm{~nm}$-sized fNDs (see Methods and Supplementary Fig. 1a), whose fluorescence stems from nitrogen-vacancy colour centres created within the nanocrystals and acting as perfectly photostable and non-blinking emitters in the far-red spectral domain ${ }^{9}$ (Supplementary Fig. 1b). Such remarkable properties have been already exploited in bio-applications requiring long-term tracking ${ }^{10}$. Moreover, fNDs have been reported to show a notably low cellular toxicity in primary neurons ${ }^{11}$.

Fig. 1a shows the different steps of the fND-tracking assay, from neuron dissociation to video acquisition. To introduce fND into neurons, we used the endocytosis mechanism ${ }^{12}$. We subsequently tracked fND-containing cargoes by pseudo-Total Internal Reflection Fluorescence (pseudo-TIRF) video microscopy at $20 \mathrm{~Hz}$ frame rate, and then made a thorough quantitative analysis of their motion. Pseudo-TIRF microscopy excites only the region of interest, a few micrometres in depth above the culture glass substrate (Supplementary Fig. 1c), leading to a high signal-to-background ratio (SBR). We determined the localization precision of the setup for different SBR, using fNDs immobilized on a glass coverslip (see Methods), and achieved a localisation precision smaller than $30 \mathrm{~nm}$ for SBR>3 (Fig. 1c). For the quantification of intraneuronal transport parameters, trajectories with more than $30 \%$ of constitutive points having a $\mathrm{SBR}<3$ were excluded. Using such a filter, we obtained a mean localization precision of $12 \mathrm{~nm}$ over all trajectories (Inset of Fig. 1c, $n=460$ trajectories from 11 experiments).

Fig. 1d displays a white-light illumination image of a main neuronal branch and its smaller branches, merged with one frame of Supplementary Video 1 showing fNDs at different locations (red spots), two of which moving in the same branch (Fig. 1e). Fig. 1f shows the kymograph of these two fNDs, highlighting the "stop-and-go" behaviour characteristic of organelle transport in neurons ${ }^{13}$. In order to characterize the fNDcontaining cargoes, we first simultaneously acquired LysoTracker-tagged lysosomes and fND using two-colour imaging (see Methods). We found a dynamical colocalisation of fNDcontaining cargoes and lysosomes in less than 5\% of moving fNDs (Supplementary 
Video 3 and Supplementary Fig. 2b). We next studied by immunofluorescence, on fixed neurons, the colocalisation between fND-containing cargoes and markers of early endosomes (EEA1 and Rab5), of recycling endosomes (Rab7), of late endosomes (Rab11) and of post-Golgi endosomes (TGN38) ${ }^{14}$. We observed that fNDs can colocalize with each type of compartments (Supplementary Fig. 2). Together, our results indicate that fNDcontaining cargoes are endosomes in a vast majority of the cases.

In order to quantify the motion of fND-containing endosomes, we first obtained the fND trajectories from the videos and identified the "stop" and "go" phases (Supplementary Fig. 3 and Fig. 1g) from which we inferred six intraneuronal transport readouts: velocity (in $\mu \mathrm{m} / \mathrm{s}$ ), run length (in $\mu \mathrm{m}$ ), processivity (in seconds), pausing time (in seconds), pausing frequency (events per minute) and the diffusion coefficient (in $\mu \mathrm{m}^{2} / \mathrm{s}$ ). Velocity of fND-containing endosome was calculated as the length of the displacement vector over time from the first to the last point of a "go" phase, whereas the curvilinear distance and time separating these two points corresponded to run length and processivity, respectively. The diffusion coefficient $D$ was calculated during the "go" phase, by fitting the mean-square displacement $(\mathrm{MSD})$ to the quadratic equation $\operatorname{MSD}(\Delta t)=v^{2} \Delta t^{2}+4 D \Delta t$, where $v$ is the velocity. According to the Stokes-Einstein law, $D$ is inversely proportional to the intracellular viscosity. During a "stop" phase, the pausing time was defined as the duration from the first to the last point, the pausing frequency was the number of "stop" phases per minute. Mean values and other statistical properties of these parameters are provided in Supplementary Table 1. Apart from the quantitative readouts, the fNDtracking assay can also provide a superresolution mapping of microtubule bundles in freely developing neuronal branches. For example, the two trajectories of Fig. 1g are separated by $\approx 100 \mathrm{~nm}$, suggesting that the two fND-containing endosomes travel along two adjacent microtubules inside the same branch. Supplementary Fig. 4 and Supplementary Video 4 show another region in which four trajectories of endosomes can be seen in the same neuronal branch, no more than $\approx 400 \mathrm{~nm}$ apart. Moreover, adjacent trajectories are separated by a distance of about $40-160 \mathrm{~nm}$, which is consistent with reported values ${ }^{8,15}$.

Intraneuronal transport occurs in both anterograde (i.e. from the soma to the distal part) and retrograde directions (from distal part to soma). In order to unambiguously measure the transport parameters associated to each direction, we cultured hippocampal neurons in a microfluidic two-chamber device to ensure the directed growth of axons (see 
Methods, Fig. 2 and Supplementary Video 5). We observed that transport parameters are identical for anterograde and retrograde direction (Fig. 2c, $p>0.05,3$ microfluidic chambers and 3 cultures, $n=22$ and $n=51$ trajectories for anterograde and retrograde direction respectively), as already reported for primary culture of hippocampal neurons ${ }^{6}$. For this reason, we applied the assay to all freely-developing branches of neurons. This approach contributed to get a high throughput in transport parameters measurements (Fig. 1b). Finally, we checked that these readouts remained constant during the 45-minute recordings for each culture well (Supplementary Fig. 5), and that there were no significant differences between the readout values for two independent experiments (Supplementary Fig. 6).

Next, as a comparison to a reference label for nanoscale tracking, we tested quantum dots with similar charge, size and fluorescence spectrum than fNDs (see Methods). We observed that QDs are less efficiently internalized (Supplementary Table 2) and that all the moving QDs display ON-OFF fluorescence blinking, indicating that there is only a single QD per cargo (see Supplementary Video 6). This ON-OFF random blinking impacts the trajectory reconstruction and leads to biased transport parameters (Supplementary Fig. 7). As a consequence, the transport parameters inferred from the QD-tracking assay were not identical for technical duplicates, contrary to fNDs (Supplementary Fig. 8 and Supplementary Table 2 for a comparison of QD and fND in term of tracking performance). Altogether, these results demonstrate that perfect photostability of fND is the key for a reliable transport measurement.

We then checked the sensitivity of the fND-tracking assay using a pharmacological drug impacting the microtubule-based intraneuronal transport, at nanomolar concentrations. This transport is dependent on the microtubule cytoskeleton integrity, and microtubule can be depolymerized by extracellular application of nocodazole. Here, we used $2 \mathrm{nM}$ and $5 \mathrm{nM}$ nocodazole concentration during the course of the $1 \mathrm{~h}$-experiment, whereas this drug is standardly applied at micromolar concentrations for several hours ${ }^{16}$. As shown in Fig. 3a ( 4 embryos, $n=39$, 33 and 40 trajectories for $0 \mathrm{nM}, 2 \mathrm{nM}$ and $5 \mathrm{nM}$ respectively), we observed (i) a decrease in velocity $\left(p=3.8 \times 10^{-4}\right.$ and $p=1.4 \times 10^{-7}$ for $2 \mathrm{nM}$ and $5 \mathrm{nM}$ respectively), in run length $\left(p=3.4 \times 10^{-4}, p=5.3 \times 10^{-6}\right)$ and in processivity $\left(p=4.5 \times 10^{-3}\right.$, $\left.p=9.8 \times 10^{-5}\right)$, and (ii) an increase in pausing time $\left(p=0.003, p=6.4 \times 10^{-5}\right)$. Conversely, we observed a decrease in pausing frequency only for $5 \mathrm{nM}(p=0.016)$ and the diffusion coefficients were not significantly affected (Supplementary Fig. 9). The increase in 
pausing time and decrease in run length and processivity are consistent with motors unable to overcome the interruptions caused by microtubule depolymerisation. The constancy of the diffusion coefficient indicates that the viscosity is not affected inside neuronal branches. Altogether, intraneuronal transport parameters vary in a drug dosage-dependent manner and change as expected for microtubule depolymerisation.

Axonal defects and intracellular transport impairments have been identified in the early stages of Alzheimer's disease both in humans and in mouse models ${ }^{17,18}$. Amyloid- $\beta$ (A $\beta$ ) peptide derived from the amyloid precursor protein together with TAU microtubuleassociated protein, a key pathogenic factor in Alzheimer's disease ${ }^{19}$, have been found to contribute to these transport deficits ${ }^{20}$. As a validation of the ability of fND-tracking assay to record abnormal intraneuronal transport, we tested $A \beta_{1-42}$ oligomers on intracellular endosome transport in cultured mouse hippocampal neurons. Here, we used $A \beta_{1-42}$ concentrations of $0.5 \mu \mathrm{M}$ and $1 \mu \mathrm{M}$ (mixture of monomer, trimer and tetramer, see Fig. 3b, 1 culture, 2 glass coverslips, $n=127,149$ and 137 trajectories for $0 \mu \mathrm{M}, 0.5 \mu \mathrm{M}$ and $1 \mu \mathrm{M}$ respectively) for $24 \mathrm{~h}$ under physiological conditions. As shown in Fig. 3c, we observed (i) an increase in pausing time ( $p=0.0014$ and $p=0.002$ for $0.5 \mu \mathrm{M}$ and $1 \mu \mathrm{M}$ respectively), and (ii) a decrease of velocity $(p=0.86, p=0.015)$, run length $(p=0.66, p=0.0003)$ and processivity $(p=0.22, p=0.0004)$. Note that the pausing frequency is decreased at concentration as low as $0.5 \mu \mathrm{M}(p=0.017)$ (Supplementary Fig. 10). The increase in pausing time and decrease in run length and processivity are consistent with previous reports on impairment of intraneuronal transport by $A \beta_{1-42^{17}}$. Moreover, transport parameters vary in a $A \beta_{1-42}$ dosage-dependent manner at submicromolar concentrations. The fND-tracking assay could therefore be used to screen drugs capable of rescuing the damaged intraneuronal transport found in AD.

Next we evaluated if the fND-tracking assay is sensitive enough to reveal the impact, on the intraneuronal transport, of slight protein concentration changes $(\approx 30 \%)$ found in brain of patients ${ }^{21,22}$. We first assessed the sensitivity of the methodology to test the change in concentration of proteins directly bound to microtubule, such as TAU or MAP2. These molecules compete with molecular motors for MT binding sites, therefore acting as roadblocks ${ }^{23}$. Genetic risk factors regulating the attachment/detachment of MAPs, such as the protein kinase coded by MARK1 gene, have long been associated to Alzheimer's disease $^{24}$ and Autism Spectrum Disorders ${ }^{21}$. We generated two Mark1 transgenic mouse lines (Fig. 4a), leading to three distinct Mark1 overexpression levels, namely line 38 
(L38 ${ }^{\text {het }}$, heterozygotes), line 8 (L8het, heterozygotes) and line 8 (L8hom, homozygotes). We first measured the protein levels of Mark1 in WT, L38 het and L8het animals (Fig. 4b, Supplementary Fig. 11a; $n=10,9$ and 9 mice for WT, L38 het and L8het respectively). L38 het and WT were not statistically different $(8 \pm 4 \%, p=0.85)$, in contrast to L8het which displayed $32 \pm 6 \%$ increase $(p=0.011)$. In comparison, most common mouse models usually have larger modifications in protein content but suffer from artificial phenotypes. We decided to test if this slight $\approx 30 \%$ increase is sufficient to induce changes in intraneuronal transport parameters, taking advantage of four different genotypes: WT, L38 ${ }^{\text {het, }}$ L8 ${ }^{\text {het }}$ and L8hom (Fig. 4c). The Mark1 gene overexpression in L8hom was measured by RT-QPCR to be twice that in L8het $\left(42 \pm 5 \%\right.$ for L8 ${ }^{\text {het }}, p=0.0013$ and $87 \pm 15 \%$ for L8hom, $p=0.0010 ; n=4,4$ and 3 cultures for WT, L8 ${ }^{\text {het }}$ and L8 $8^{\text {hom }}$ respectively). This measurement was done on the same primary cultures as the ones used for the fND-tracking assay, right after trajectories acquisitions.

Quantification of the intraneuronal transport dynamics revealed significant variations for L8het and L8hom, but not for L38 het in which no changes were observed in any of the readouts (Fig. 4c, Supplementary Fig. 11b; $n=420$ trajectories/10 cultures, $n=62 / 4$, $n=145 / 4$, and $n=85 / 3$ for WT, L38 ${ }^{\text {het }}$, L8 ${ }^{\text {het }}$ and L8 ${ }^{\text {hom }}$ respectively). The latter is consistent with the absence of Mark1 overexpression for this transgenic line, therefore acting as an internal control. In L8het and L8hom, the monotonous increase in Mark1 gene overexpression levels was accompanied by a corresponding increase in velocity $(p=0.03$, $p=0.006$ for L8 ${ }^{\text {het }}$ L $8^{\text {hom }}$ respectively), in run length ( $p=0.029, p=0.022$ for L8 ${ }^{\text {het }}$, L8 ${ }^{\text {hom }}$ respectively) and in processivity ( $p=0.037$ for L $8^{\text {hom }}$ respectively). We also observed a decrease in pausing frequency for L8 ${ }^{\text {het }}(p=0.044$, Supplementary Fig. 11b). Altogether, intraneuronal transport parameters vary in a gene dosage-dependent manner and they change as expected for Mark1-mediated regulation of the microtubule track hindrance (Fig. 4d) ${ }^{24}$. We therefore demonstrated the sensitivity of our assay to detect microtubule roadblock impairments linked to brain diseases, for protein concentration changes as small as $\approx 30 \%$.

Finally, we evaluated if the fND-tracking assay is sensitive enough to detect the effect of concentration change of one protein that do not belong to microtubule complexes but is indirectly involved in the intraneuronal transport via energy supplies. We focused on the psychiatric risk factor $S L C 25 A 12$ that is required for the mitochondrial integrity and found associated with different types of Autism Spectrum Disorders and with 
Schizophrenia ${ }^{25}$. We generated one Slc25a12 transgenic mouse line (L2 ${ }^{\text {het }}$ ) mimicking the slight overexpression of this gene as found in brain tissues of Autism Spectrum Disorders patients $^{22,26}$ (Fig. 5a). We measured Slc25a12 protein levels in WT and L2 ${ }^{\text {het }}$ and found a significant increase in L2 ${ }^{\text {het }}(32 \pm 7 \%, p=0.043 ; n=5$ mice) (Fig. 5b, Supplementary Fig. 12). The quantification of the intraneuronal transport dynamic revealed significant variations with a decrease in run length $(p=0.022)$ and processivity ( $p=0.012)$ (Fig. 5c; $n=420$ trajectories $/ 10$ cultures and $n=105 / 3$ for WT and L2 ${ }^{\text {het }}$ respectively). This result demonstrates the sensitivity of the tracking assay to detect indirect intraneuronal transport perturbations, for protein concentration changes as small as $\approx 30 \%$.

In this Letter, we have reported a hitherto unexplored approach to measure the changes of intraneuronal transport parameters induced by brain disease-related genetic risk factors. Our methodology relies on fluorescent nanodiamond tracking inside freely developing branches of living neurons. We showed that the high brightness, perfect photostability and absence of cytotoxicity of fNDs make it possible to perform a high throughput and high content intracellular transport measurement based on trajectory analysis. In Supplementary Table 3 and Supplementary Text 1, we compared our fNDtracking assay to other reported methodologies used for assessing intraneuronal transport. These published techniques, based on tracking of endosomes labelled with QDs $^{8}$, lipophilic dyes ${ }^{27}$ or chimeric fluorescent proteins ${ }^{6}$, yielded a smaller number of readouts (velocity and processivity) and showed smaller spatio-temporal resolution. Moreover, we proved that unstable emission of QDs leads to unreliable measurements (Suppl. Fig. 8). The fND tracking assay has some limitations too, in particular (i) no particular cargo labelling, while chimeric fluorescent proteins can specifically tag the compartments of interest, and (ii) low two-photon absorption cross-section, preventing tracking in thick structures or in vivo by multiphoton microscopy. However, the fND nanoparticle-based tracking assay can be extended to non-linear nanolabels ${ }^{28}$.

As a proof-of-principle demonstration of fND-tracking assay unique possibilities, we established transgenic animals mimicking the changes of protein concentrations $(\approx 30 \%)$ found in brain of patients. The fND-tracking assay was sensitive enough to detect modifications of the intraneuronal transport parameters in the transgenic neurons. To the best of our knowledge, this is the first direct measurement of the functional impact of brain disease-related genetic risk factors using a nanoparticle-based methodology. 
In a near future, with the swift development of the CRISPR/Cas9 genome editing technology 29 , it is possible to model multiple brain disease-related genetic risk factors in transgenic animals. Our fND tracking methodology is adapted to understand how these multiple genetic variants interact with each other to impair the intraneuronal transport. Furthermore, powerful methodologies to dissect the molecular basis of complex brain diseases are emerging such as 3D-human neuron cultures derived from induced pluripotent stem cells obtained from patients ${ }^{30,31}$. In the long run, this method can be instrumental to develop an unbiased diagnosis of neuropsychiatric and neurodegenerative diseases, with potential applications in translational nanomedicine. 


\section{References}

1. McClellan, J. \& King, M.-C. C. Genetic heterogeneity in human disease. Cell 141, 210-7 (2010).

2. Hirokawa, N., Niwa, S. \& Tanaka, Y. Molecular motors in neurons: transport mechanisms and roles in brain function, development, and disease. Neuron 68, 610-38 (2010).

3. Millecamps, S. \& Julien, J.-P. Axonal transport deficits and neurodegenerative diseases. Nat. Rev. Neurosci. 14, 161-76 (2013).

4. Schirhagl, R., Chang, K., Loretz, M. \& Degen, C. L. Nitrogen-Vacancy Centers in Diamond: Nanoscale Sensors for Physics and Biology. Annu. Rev. Phys. Chem. 65, 83-105 (2014).

5. de Angelis, M. H. et al. Analysis of mammalian gene function through broad-based phenotypic screens across a consortium of mouse clinics. Nat. Genet. 47, 969-978 (2015).

6. Kwinter, D. M., Lo, K., Mafi, P. \& Silverman, M. A. Dynactin regulates bidirectional transport of dense-core vesicles in the axon and dendrites of cultured hippocampal neurons. Neuroscience 162, 1001-1010 (2009).

7. Pinaud, F., Clarke, S., Sittner, A. \& Dahan, M. Probing cellular events, one quantum dot at a time. Nat. Methods 7, 275-285 (2010).

8. Mudrakola, H. V, Zhang, K. \& Cui, B. Optically Resolving Individual Microtubules in Live Axons. Structure 17, 1433-1441 (2009).

9. Chang, Y.-R. et al. Mass production and dynamic imaging of fluorescent nanodiamonds. Nat. Nanotechnol. 3, 284-288 (2008).

10. $\mathrm{Wu}, \mathrm{T}$. et al. Tracking the engraftment and regenerative capabilities of transplanted lung stem cells using fluorescent nanodiamonds. Nat. Nanotechnol. 8, 682-689 (2013).

11. Huang, Y.-A. et al. The effect of fluorescent nanodiamonds on neuronal survival and morphogenesis. Sci. Rep. 4, 6919 (2014).

12. Blanpied, T., Scott, D. \& Ehlers, M. Dynamics and regulation of clathrin coats at specialized endocytic zones of dendrites and spines. Neuron 36, 435-449 (2002).

13. Overly, C. C., Rieff, H. I. \& Hollenbeck, P. J. Organelle motility and metabolism in axons vs dendrites of cultured hippocampal neurons. J. Cell Sci. 109, 971-980 (1996).

14. Rodriguez-Boulan, E., Kreitzer, G. \& Müsch, A. Organization of vesicular trafficking in epithelia. Nat. Rev. Mol. Cell Biol. 6, 233-247 (2005).

15. Chen, J., KANAI, Y., Cowan, N. J. \& HIROKAWA, N. Projection Domains of Map2 and Tau Determine Spacings Between Microtubules in Dendrites and Axons. Nature 360, 674-676 (1992).

16. Courty, S., Luccardini, C., Bellaiche, Y., Cappello, G. \& Dahan, M. Tracking Individual Kinesin Motors in Living Cells Using Single Quantum-Dot Imaging. Nano Lett. 6, 1491-1495 (2006).

17. Stokin, G. B. et al. Axonopathy and transport deficits early in the pathogenesis of 
Alzheimer's disease. Science 307, 1282-8 (2005).

18. Encalada, S. E. \& Goldstein, L. S. B. Biophysical Challenges to Axonal Transport: Motor-Cargo Deficiencies and Neurodegeneration. Annu. Rev. Biophys. 43, 141169 (2014).

19. Querfurth, H. W. \& LaFerla, F. M. Alzheimer's disease. N. Engl. J. Med. 362, 329-44 (2010).

20. Vossel, K. A. et al. Tau Reduction Prevents A -Induced Defects in Axonal Transport. Science 330, 198-198 (2010).

21. Maussion, G. et al. Convergent evidence identifying MAP/microtubule affinityregulating kinase 1 (MARK1) as a susceptibility gene for autism. Hum. Mol. Genet. 17, 2541-51 (2008).

22. Lepagnol-Bestel, A. M. et al. SLC25A12 expression is associated with neurite outgrowth and is upregulated in the prefrontal cortex of autistic subjects. Mol. Psychiatry 13, 385-97 (2008).

23. Lakadamyali, M. Navigating the cell: how motors overcome roadblocks and traffic jams to efficiently transport cargo. Phys. Chem. Chem. Phys. 16, 5907-16 (2014).

24. Mandelkow, E.-M. E., Thies, E., Trinczek, B. \& Biernat, J. MARK/PAR1 kinase is a regulator of microtubule-dependent transport in axons. J. Cell Biol. 167, 99-110 (2004).

25. Fromer, M. et al. De novo mutations in schizophrenia implicate synaptic networks. Nature 506, 179-84 (2014).

26. Palmieri, L. et al. Altered calcium homeostasis in autism-spectrum disorders: evidence from biochemical and genetic studies of the mitochondrial aspartate/glutamate carrier AGC1. Mol. Psychiatry 15, 38-52 (2010).

27. Nakamura, H. et al. Quantitative analysis of intraneuronal transport in human iPS neurons. J. Pharmacol. Sci. 128, 170-178 (2015).

28. Mayer, L. et al. Single KTP nanocrystals as second-harmonic generation biolabels in cortical neurons. Nanoscale 5, 8466-71 (2013).

29. Doudna, J. A. \& Charpentier, E. The new frontier of genome engineering with CRISPR-Cas9. Science 346, 1258096-1258096 (2014).

30. Choi, S. H. et al. A three-dimensional human neural cell culture model of Alzheimer's disease. Nature 515, 274-278 (2014).

31. Paşca, A. M. et al. Functional cortical neurons and astrocytes from human pluripotent stem cells in 3D culture. Nat. Methods 12, 671-678 (2015). 


\section{Acknowledgments}

We thank Paul Tchénio for his comments on the manuscript, Alain Lombard for his contribution to data processing, Joanna Lipecka for her help with western blotting. This work was supported by a public grant overseen by the French National research Agency (ANR) as part of the "Investissement d'Avenir » program IDEX Paris-Saclay ANR-11IDEX- 0003-02 (S.H.); the ANR and the Ministry of Science and Technology (Taiwan) through grant ANR-2010-INTB-1002 (M.S., F.T., H.C.C., C.C.W.); the ANR through the Eranet Euronanomed project 2011-ERNA-006 (F.T., M.S., C.S., A.C.); the Fondation Jérôme Lejeune (M.S.), the European Union FP7-Health "AgedBrainSysBio" grant (M.S.); grant $\mathrm{n}^{\circ} 12018592$ from Region Ile-de-France (F.T.); CNRS "Programme interdisciplinaire” (F.T., M.S.) and Triangle de la Physique contract no. 2012-038T (F.T., M.S.).

\section{Authors contributions}

SH, FT and MS conceived and designed the experiments; SH, NM, YLM, AMLB, MPA, SM, XLL, JV, CP, ED, CR and RD performed the experiments; SH, NM, SM, MPA and XLL analyzed the data; PK, FJH, CCW, BP, YH, CS, AC, BA and HCC contributed to materials/analysis tools; $\mathrm{SH}, \mathrm{FT}$ and MS wrote the paper.

\section{Additional information}

Supplementary information is available in the online version of the paper. Reprints and permissions information is available online at www.nature.com/reprints. Correspondence and requests for materials should be addressed to F.T. or M.S.

\section{Competing financial interests}

The authors declare no competing financial interests. 


\section{Figures}
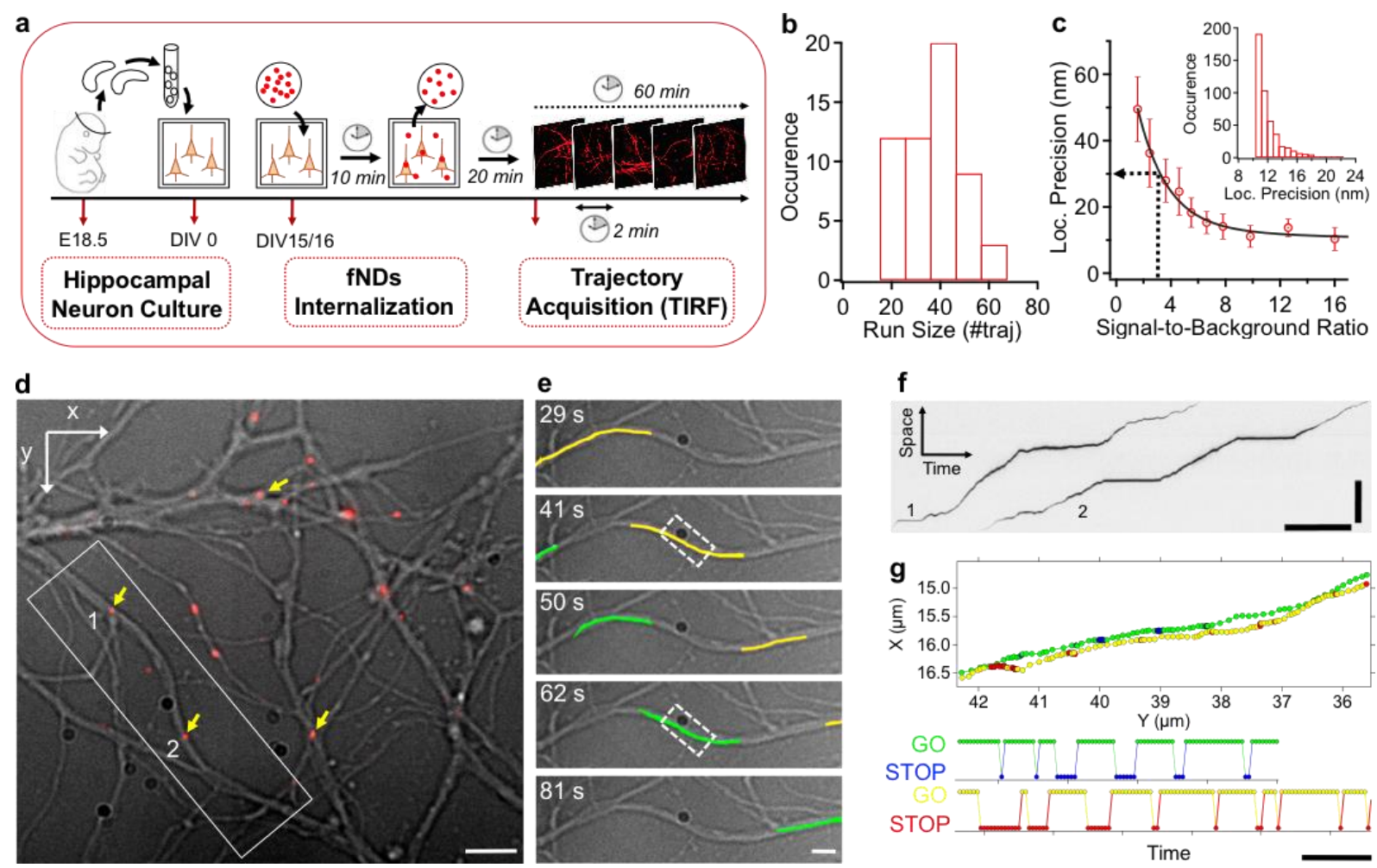

Figure 1 | Intraneuronal transport monitoring by fluorescent nanodiamond tracking. fNDs internalise spontaneously and efficiently in neuronal branches. Their tracking by fluorescence video-microscopy at high spatio-temporal resolution allows to reconstruct their trajectories. STOP and GO phases detection make it possible to extract several transport parameters. a, Experimental pipeline from hippocampal neuron culture dissociated from E18.5 mouse embryo to endosome trajectory acquisition using pseudoTIRF microscopy. DIV: day in vitro. b, Histogram of dataset sizes, i.e. number of trajectories exploited for readouts quantification. The mean value is 37 trajectories/run ( $n=56$ runs). c, Localization precision versus signal-to-background ratio (SBR) of fNDs immobilized on a glass coverslip (from 100 fNDs imaged over 200 frames; data plotted as mean+/standard deviation). By applying a cut-off at $\mathrm{SBR}=3$, we obtained a localization precision better than $30 \mathrm{~nm}$. Inset: distribution of the mean localization precision for each fNDcontaining endosome trajectory ( $n=460$ trajectories from 11 wild type experiments). d, Transmission white-light illumination image of the neuronal branches merged with the fluorescence channel (extracted from Supplementary Video 1) showing four fNDs moving within dendrites (yellow arrows). The cell soma associated with the main branch is on the left (not visible). During the two-minute movie, two fND-containing endosomes, labelled \#1 and \#2 (solid white line box) were observed moving towards the cell soma, in the same branch. e, Superimposition onto a white light image of the positions of these two fNDs (\#1, in yellow; \#2 in green), determined by particle tracking, with a persistence of $10 \mathrm{~s}$, at different times. Scale bars: $5 \mu \mathrm{m}$ in (d) and $1 \mu \mathrm{m}$ in (e). f, Kymograph of the motion of fNDs \#1 and \#2 shown in (e)(white box), illustrating their "stop-and-go" behavior. Scale bars: $15 \mu \mathrm{m}$ (space) and $10 \mathrm{~s}$ (time). g, Top: trajectories of fND \#1 and \#2 extracted from the 
fluorescence video (dashed line rectangle in (e)). The two colours in each trajectory correspond to "go" phases (in green for fND \#1 and yellow for fND \#2) and "stop" phases (in blue for fND \#1 and red for fND \#2), as identified by our trajectory analysis algorithm (see Methods); bottom: two-state ("stop" and "go") representation of the same trajectories over time: fND \#1 moved faster than fND\# 2 (scale bar: $1 \mathrm{~s}$ ).

a

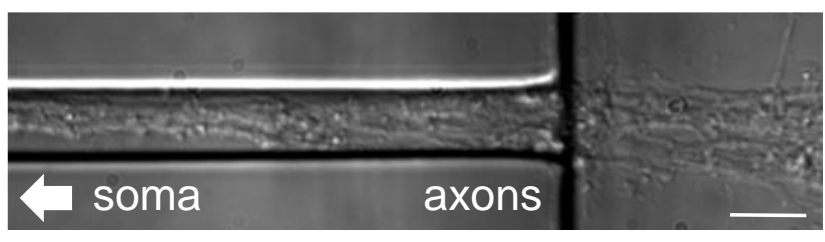

b
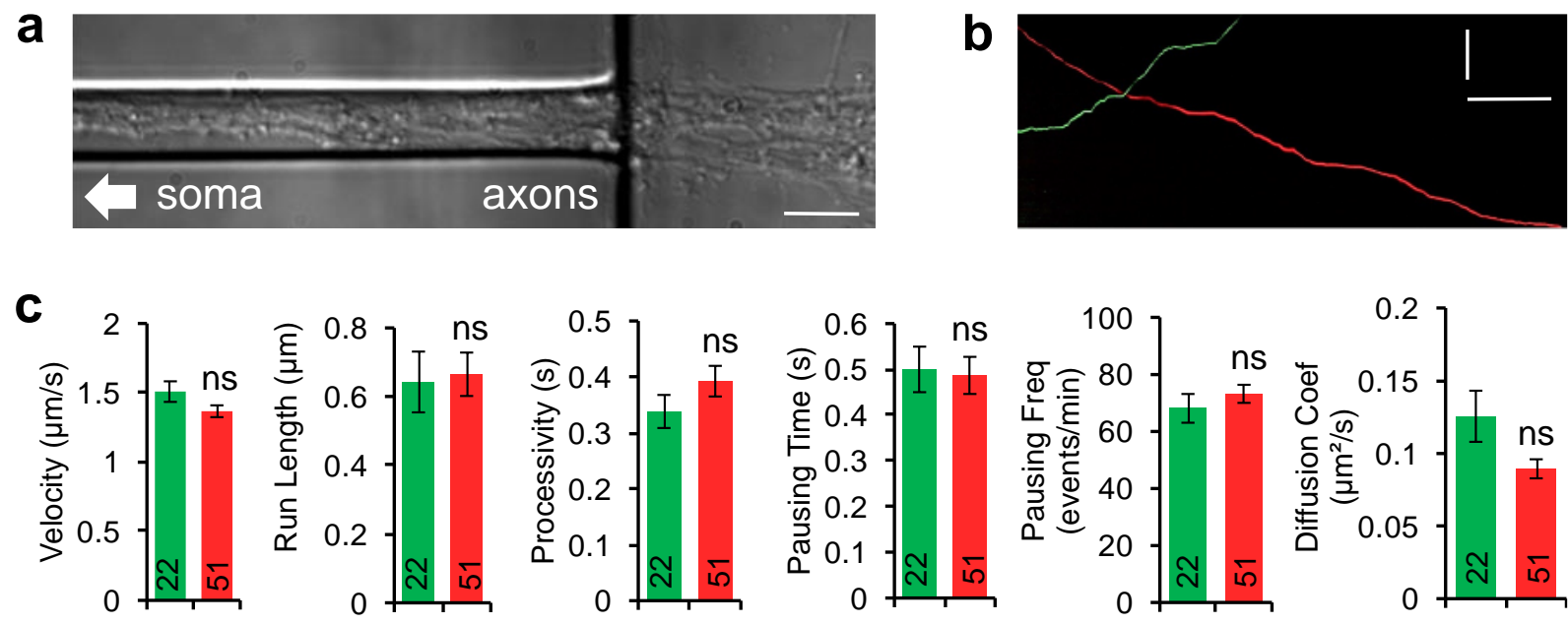
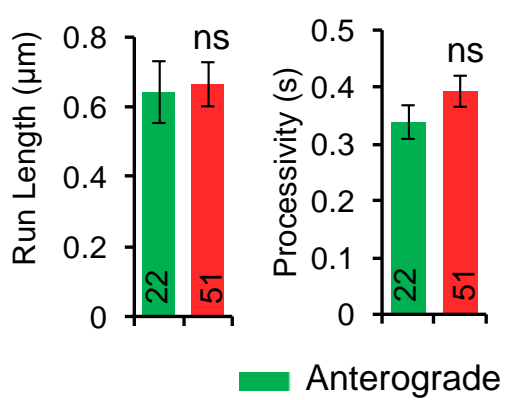
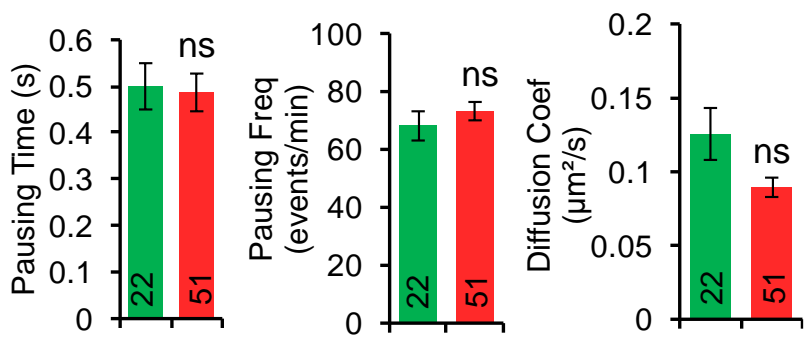

Retrograde

Figure 2 | Transport parameter quantification for anterograde and retrograde motions in axons of neurons grown in microfluidic chamber. a, DIC image of axons emerging from one microfluidic channel. Scale Bar: $5 \mu \mathrm{m}$. b, Colour-coded kymograph showing anterograde (green) and retrograde (red) motions. Scale bars: time (horizontal) $5 \mathrm{~s}$, space (vertical) $10 \mu \mathrm{m}$. c, Quantification of the transport readouts. No significant (ns) differences between both directions of motion were observed (Wilcoxon and MannWhitney two-tailed test $p>0.05$ ). Data are displayed as mean values \pm s.e.m. Figures written inside the bars represent the total number of trajectories from $n=3$ microfluidic chambers from $n=3$ cultures. 

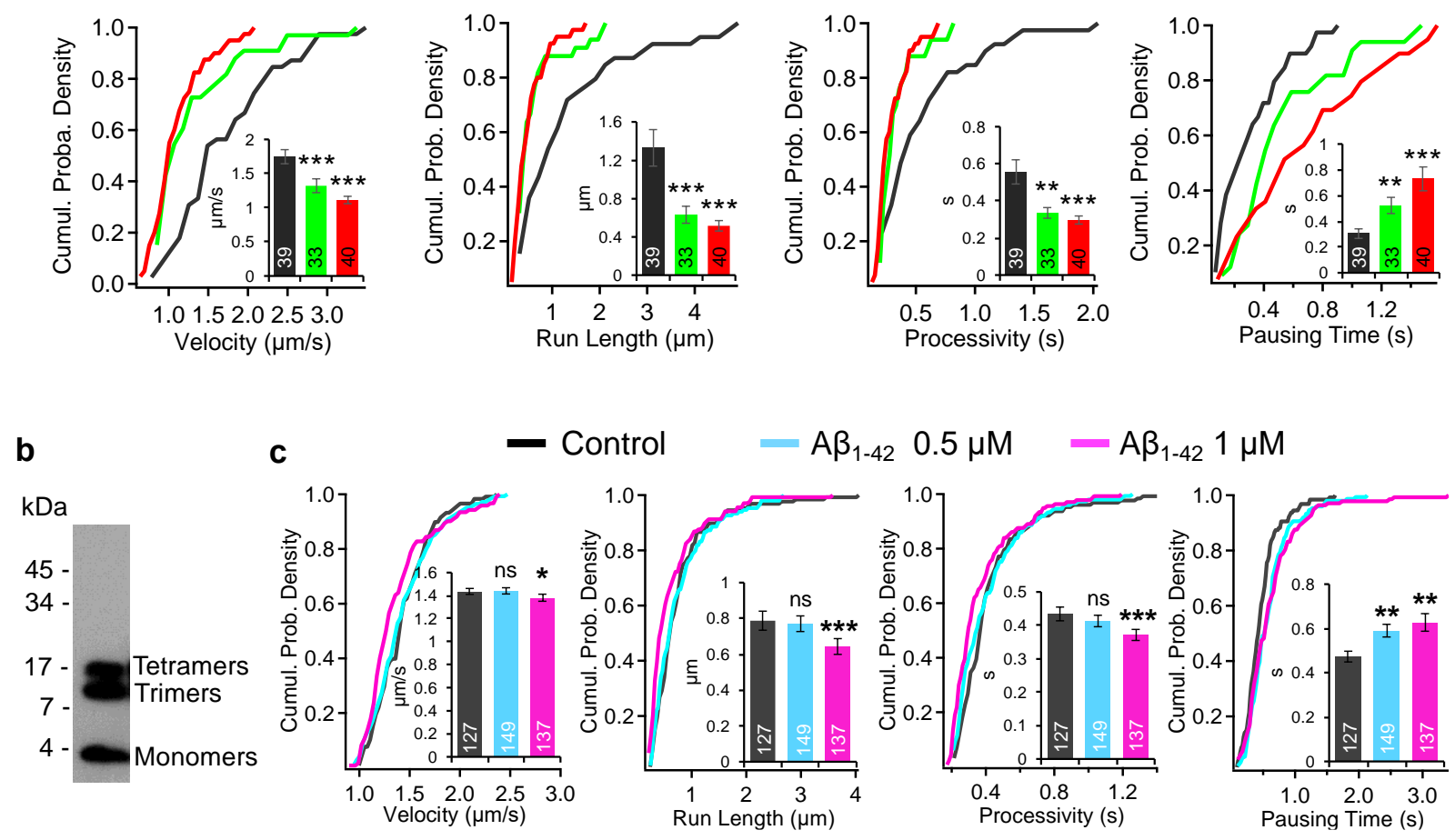

Figure 3 | Effect of nocodazole and Alzheimer's disease-related A $\beta_{1-42}$ oligomers on the intraneuronal transport. a, For nocodazole, significant changes of velocity, run length, processivity and pausing time are detected at concentration as small as $2 \mathrm{nM}$ (green). These changes are stronger at $5 \mathrm{nM}$ (red). b, Western blot of $\mathrm{A} \beta_{1-42}$ preparation showing the presence of monomers, trimers and tetramers. c, $A \beta_{1-42}$ used at concentrations of $0.5 \mu \mathrm{M}$ (blue) and $1 \mu \mathrm{M}$ (purple) induce an increase in pausing time concentration as small as $0.5 \mu \mathrm{M}$ and significant changes of all parameters at $1 \mu \mathrm{M}$. Figures written inside the bars represent the total number of trajectories analysed from $n=2$ glass coverslips. Data are displayed as cumulative probabilities and bar plots with mean values \pm s.e.m in insets. Statistical significance: one star $\left(^{*}\right)$ indicate a $p$ value smaller than 0.05 (Wilcoxon and Mann-Whitney two-tailed test), two stars (**) indicate a $p<0.01$ and three stars $\left.{ }^{* * *}\right)$ mean that $p<0.001$. 


\section{a}

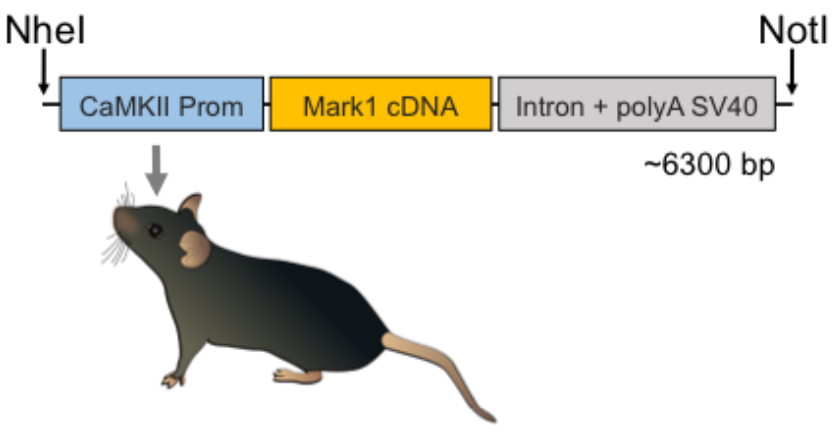

b

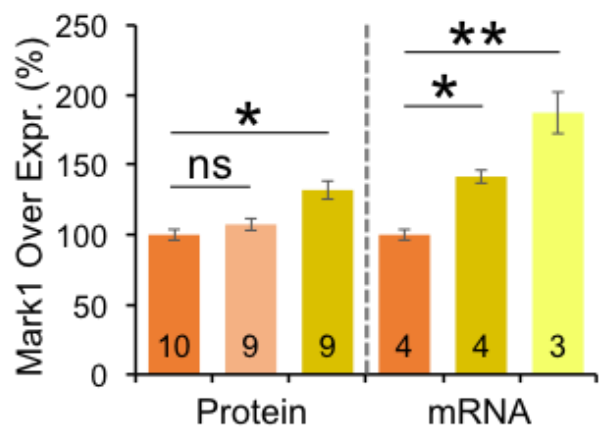

C
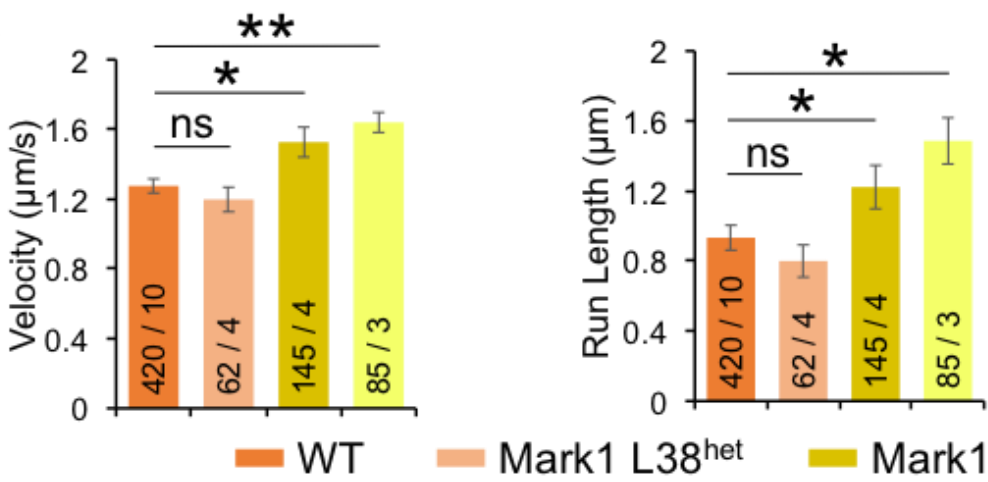

Mark1 L38

Mark1 L8 ${ }^{\text {het }}$

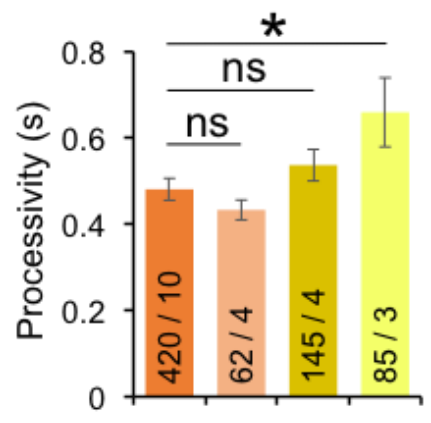

Mark1 L8 hom

d
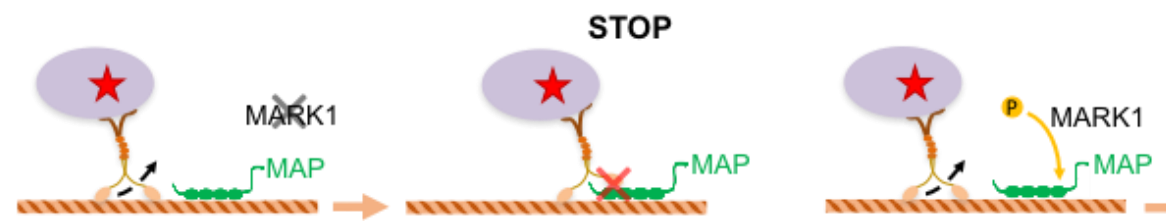

GO

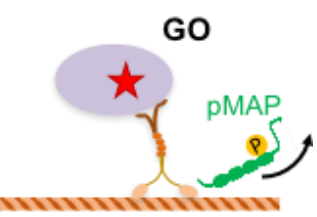

Figure 4 | Sensitivity of the fND-tracking assay to slight concentration changes of autism-related Mark1 protein. a, Schematic representation of the Mark1 transgene used to create transgenic mouse L38 and L8. b, Quantification of Mark1 gene overexpression levels. The protein levels of Mark1 in WT, L38 het and L8 $8^{\text {het }}$ animals were measured by Western Blot. Mark1 overexpression in WT, L8het and L8hom was evaluated by RT-QPCR (same neurons as the ones used in (c)). L8 $8^{\text {het }}$ and L8 ${ }^{\text {hom }}$ display a significant increase of Mark1 expression, but not L38 ${ }^{\text {het. }}$ c, Effects of Mark1 overexpression on velocity, run length and processivity intraneuronal transport parameters, revealing significant variations for L8 ${ }^{\text {het }}$ and L8 ${ }^{\text {hom }}$, but not for L38 ${ }^{\text {het. }}$. , Mechanistic model of the impact of Mark1 protein on intraneuronal transport, as described in Mandelkow et. al. ${ }^{24}$ : at normal concentration of MARK1, microtubule associated proteins MAP are bound to the microtubule and act as roadblocks of endosomal transport. However, when MARK1 kinase is in excess, the phosphorylated forms of MAP that does not bind to microtubules are more abundant, which reduce the amount of roadblocks and facilitate the endosomal transport as observed by the increases of velocity and run length. Such mechanism is consistent with our data. All data are presented as mean \pm s.e.m. (calculated from the total number of samples in (b) or of independent cultures in (c)). Figures written inside the bars represent the number of samples (mouse tissue for proteins and neuron cultures for mRNA) in (b) or the number of trajectories/number of independent cultures in (c). One experimental run is associated to one culture of one embryo. Statistical significance: one 
star $\left({ }^{*}\right)$ indicates $p$ value smaller than 0.05 (Wilcoxon and Mann-Whitney two-tailed test), two stars $(* *)$ are for $p<0.01$.

a

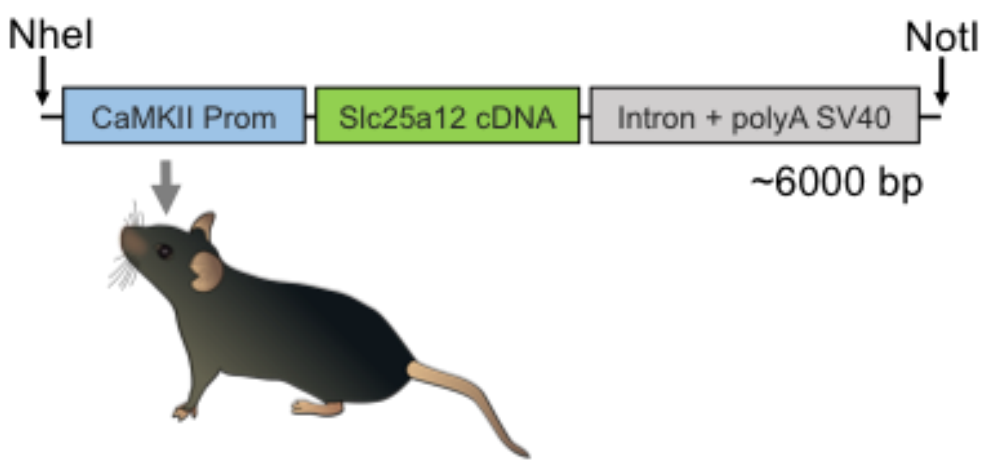

C

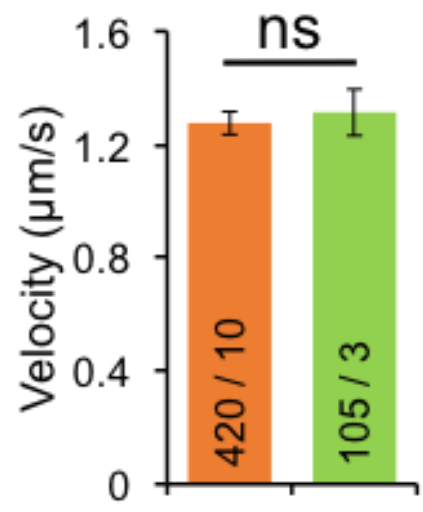

WT
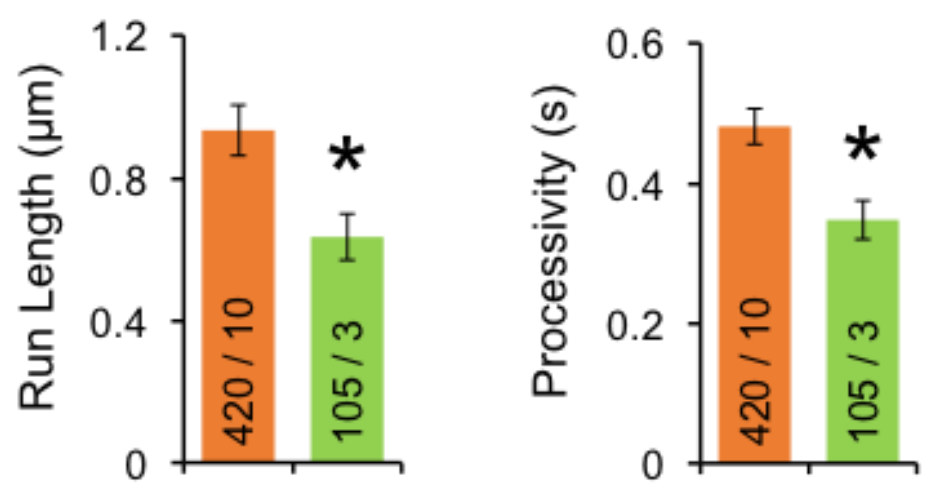

Figure 5 | Sensitivity of the fND-tracking assay to slight overexpression of autismrelated Slc25a12. a, Schematic representation of the Slc25a12 transgene used to create transgenic mouse line L2. b, Quantification of Slc25a12 expression in L2 ${ }^{\text {het }}$ showing an increase of $32 \pm 7 \%$ ( $p=0.043$ ) in Slc25a12 protein level as measured by Western Blot. c, Effects of Slc25a12 overexpression on velocity, run length and processivity intraneuronal transport parameters. Velocity and run length are decreased when Slc25a12 is slightly overexpressed. All data are presented as mean \pm s.e.m. (calculated from the total number of samples in (b) or of independent cultures in (c)). Figures written inside the bars represent the number of mouse hippocampus in (b) or the number of trajectories/number of independent cultures in (c), with one experimental run associated to one culture of one embryo. Statistical significance: one star $\left({ }^{*}\right)$ indicates $p$ value smaller than 0.05 (Wilcoxon and Mann-Whitney two-tailed test). 


\section{Methods}

\section{Fluorescent nanodiamond production.}

The nanodiamonds were radiation-damaged with a high-energy ( $3 \mathrm{MeV}$ ) proton beam, as detailed in Su et al. $^{32} \mathrm{~A}$ thin diamond film (thickness $<50 \mu \mathrm{m}$ ) was prepared by depositing $\approx 5 \mathrm{mg}$ of nanodiamond powder on a silicon wafer $\left(1 \times 1 \mathrm{~cm}^{2}\right.$ size $)$ and subsequently subjected to the ion irradiation at a dose of $\approx 2 \times 10^{16} \mathrm{H}^{+} . \mathrm{cm}^{-2}$. Afterwards, the radiationdamaged nanodiamonds were annealed at $800^{\circ} \mathrm{C}$ for $2 \mathrm{~h}$ to form fNDs. To remove graphitic carbon atoms on the surface, the freshly prepared fNDs were oxidized in air at $490^{\circ} \mathrm{C}$ for $2 \mathrm{~h}$ and microwave-cleaned in concentrated $\mathrm{H}_{2} \mathrm{SO}_{4}-\mathrm{HNO}_{3}(3: 1, \mathrm{v} / \mathrm{v})$ at $100^{\circ} \mathrm{C}$ for $3 \mathrm{~h}$. The average number of nitrogen vacancy colour centres per fND is 15 .

\section{Pseudo-TIRF Microscopy setup and video acquisition}

TIRF illumination was implemented on a two-stages inverted microscope (Eclipse Ti-E, Nikon, Japan), equipped with 100x magnification and 1.49 numerical aperture immersion oil objective (CFI Apo TIRF 100X Oil, Nikon, Japan). The excitation beam from a continuous-wave diode-pumped solid-state laser emitting at the wavelength of $561 \mathrm{~nm}$ with a maximum power of $100 \mathrm{~mW}$ (SLIM-561-100, Oxxius S.A., France) was expanded $16 \mathrm{x}$ and then focused on the back focal plane of the microscope objective, off the propagation axis to ensure partial internal reflection at the culture dish glass - medium interface. This configuration results in wide-field illumination of a volume confined to $\approx 2 \mu \mathrm{m}$ above this interface and to an in-plane disc of $\approx 40 \mu \mathrm{m}$ diameter, ensuring a larger signal-to-background ratio than in epifluorescence configuration. The maximum laser intensity was $20 \mathrm{~kW} / \mathrm{cm}^{2}$ in the sample plane. We used filters adapted to the spectral characteristics of nitrogen vacancy colour centres in diamond: a dichroic beamsplitter with a steep edge at $561 \mathrm{~nm}$ and a 97\% flat transmission above this wavelength (zt561rdc, Chroma Corp., USA) and a band-pass filter centred on $697 \mathrm{~nm}$ wavelength with a fullwidth at half maximum (FWHM) of 75 nm (HC 697/75, Semrock, USA), on the detection channel. White-light illumination observations were done with the differential interference contrast mode of the microscope without changing the microscope objective. The whole microscope was enclosed in a cage incubator (Okolab, Italy) allowing to maintain a temperature of $37^{\circ} \mathrm{C}, 5 \%$ partial $\mathrm{CO}_{2}$ pressure and $100 \%$ hygrometry, necessary for live neuron imaging. Images and videos were recorded with a cooled electron multiplied CCD array detector (iXon-DU885, Andor Technology, Ireland), having a pixel size in the sample plane of $80 \mathrm{~nm}$.

\section{Video processing, trajectory extraction and analysis.}

We recorded videos of total duration of $120 \mathrm{~s}$, at a time resolution of $50 \mathrm{~ms} /$ frame with Nikon NIS software in the proprietary ND2 format (Fig. 1a). A filtering pretreatment was done with Fiji software (version 1.46j; NIH, USA), which consists in subtracting to each frame the median intensity of the whole stack in order to remove static fluorescent spots. For each dynamic fND-containing endosome, we reconstructed the full trajectory using the Spot Detector plugin of ICY image analysis software (GPLv3 open source) developed by the Quantitative Image Analysis team at Institut Pasteur (Paris, France) ${ }^{33}$. Using the Track Manager plugin, we exported a text file containing the spot $(X, Y)$ coordinates and the signal amplitude and background (based on a 2D-gaussian fitting of fluorescence spots), and we processed these data with a home-made program written for Igor Pro 
software (WaveMetrics Inc., USA). Detection of "stop" and "go" phases relies on two main steps, namely a coarse "stop" phase detection based on the confinement ratio function, and a refine "go" detection based on three different sequential filters as detailed in Supplementary Fig. 3. Transport readouts for each trajectory are then computed and the program finally makes a statistical report for the entire run set corresponding to one experiment, namely one LabTek well. The IgorPro program is available upon request.

Localization precision. We calibrated the localization precision of our set-up by tracking the position of more than 100 fNDs immobilized on a glass coverslip and imaged over 100 frames. We then analysed the video with the same tracking software parameters as for the intraneuronal trajectories. Movies were background-subtracted using Fiji plugin with a rolling ball of 20 pixels, in order to mimic the effect of a median subtraction. For each fND, the localization precision was measured as the standard deviation of the center positions of the fluorescent spot over time. We then plotted all localization precisions with respect to the signal-to-background ratio (SBR) associated to each fND (Fig. 1c). SBR was determined as the ratio of the maximum fluorescence intensity of the fND (amplitude minus residual background) over the residual background intensity. Therefore, trajectories containing more than $30 \%$ of points having a SBR smaller than 3 were not considered, so that according to the calibration, we achieved a localization precision better than $30 \mathrm{~nm}$.

\section{Primary hippocampal neuron cultures.}

For wild type neuron cultures, hippocampi from E18.5 OF1 mouse embryos were isolated in a dissecting medium (HBSS-Hepes) and hippocampus from at least four embryos were pooled together. Hippocampal neurons were then enzymatically dissociated $(0.25 \%$ trypsin), mechanically triturated and plated on LabTek II (Nunc, USA) dishes $\left(2 \times 10^{5}\right.$ cells per well) coated with poly-DL-ornithin (Sigma-Aldrich, USA), in DMEM (Invitrogen, USA) supplemented with 10\% foetal bovin serum (FBS), 0.1\% GlutaMAX (Invitrogen) and 1\% PenStrep (Invitrogen). Two hours after plating, DMEM was replaced by Neurobasal phenol-free medium (Invitrogen) supplemented with 0.1\% GlutaMAX (Invitrogen), 2\% B27 (Invitrogen) and 1\% PenStrep (Invitrogen). Cultures from C57Bl6 transgenic mouse were conducted separately for each embryo and genotyping was performed elsewhere. Cultures in microfluidic devices (RD450 from Xona Microfluidics, USA) were performed on coated $22 \mathrm{~mm}$ coverslip, plated with $5.10^{6}$ cells/chambers. Neurons were grown until DIV $17 / 18$.

\section{fNDs internalization in hippocampal neuron cultures.}

We conducted fNDs internalization in neurons at DIV15/16. The maintaining medium was set aside and replaced by $0.5 \mathrm{ml}$ of Neurobasal phenol-free medium (Invitrogen) to which we added $5 \mu \mathrm{l}$ of $\approx 30 \mathrm{~nm}$-sized fND stock solution, reaching a final concentration of $5 \mu \mathrm{g} / \mathrm{ml}$. After $10 \mathrm{~min}$ incubation, the medium was replaced by the old maintaining medium and neurons were placed $20 \mathrm{~min}$ in the incubator before starting the video acquisition. The same internalization procedure was applied to QD (ref. Q21361MP from ThermoFisher, USA) but with 1:10 5 dilution of the stock solution i.e. $0.1 \mathrm{nM}$ final.

\section{fND-containing cargos staining and colocalisation analysis.}


For lysosomes labelling, live neurons were stained using LysoTracker Green DND-26 (ref. L7526, ThermoFisher) applied at $100 \mathrm{nM}$ concentration at least $1 \mathrm{~h}$ prior to acquisition. We only considered internalized fNDs, i.e. having either a directed motion or a static colocalisation with a lysosome (Supplementary Fig. 2b). For endosomes immunostaining, neurons were fixed with $4 \%$ PFA for $20 \mathrm{~min}$ at room temperature. EEA1, Rab5, Rab11, Rab7 and TGN38 were labelled overnight at $4^{\circ} \mathrm{C}$ using Cell Signaling (USA) ref. C45B10, C8B1 and D4F5, and Santa Cruz (USA) Ref. sc-271608, sc-271624 respectively as primary antibodies. Secondary antibodies tagged with AlexaFluor 488 dye (Ref. 111-545-144 and 715-547-003, from Jackson ImmunoResearch Inc., USA) were then applied for $1 \mathrm{~h}$ at room temperature. Fluorescence acquisitions were performed in two colours with the same electron multiplied CCD array detector array as for videomicroscopy, with a DualView DV2 system placed in front of the detector (Photometrics, USA). The DualView splits the image vertically in two parts: on one half of the sensor surface green light emitted by LysoTracker and AlexaFluor 488 is detected, and red light from fNDs is recorded on the other half (Supplementary Video 3). Fluorophores are excited with solid-states lasers: Sapphire 488 (Coherent, USA) at $488 \mathrm{~nm}$ wavelength for LysoTracker and AlexaFluor 488, and SLIM 561 (Oxxius S.A., France) at $561 \mathrm{~nm}$ wavelength for fNDs. The two laser beams are combined with a dual-band dichroic filter (Ref. Di01-R488/561, from Semrock, USA). The DualView contains another green/red dichroic filter followed on each channel by a bandpass filter (Ref. D535/40M for green emitters and ET-BandPass-700-75 for fND, both from Chroma Technology, USA). The excitation laser powers were adjusted for each channel so that dynamic range was identical at given camera settings (EMgain $=60$, preamp gain $=3.8$, and digitalization speed $35 \mathrm{MHz}$ ). The slight shift between both channels was measured using $100 \mathrm{~nm}$ TetraSpeck fluorescent beads (Ref. T7279, ThermoFisher). For colocalisation study of fixed cultured, $z$-stack (7 planes with $0.25 \mu \mathrm{m}$ step, total of $1.75 \mu \mathrm{m}$ displacement) were recorded for each field of view and maximum intensity projections were carried out. As for video microscopy, we used ICY Spot Detector ${ }^{33}$ plugin to extract spot coordinates (in pixels), spot maximum intensity (in counts) and spot size (surface in pixels\#). Then, a custom program was written with IGOR Pro to count colocalised spots between each half of the image within 1.5 pixels uncertainty (equivalent to one standard deviation) in $x$ and $y$ directions. To filter out non-specific labelling, we only considered spots composed of 15 pixels $(\approx 350 \mathrm{~nm}$ in diameter, compatible with the convolution of the microscope point spread function and $100 \mathrm{~nm}$ sized endosomes) and being at least three times brighter than the dimmer spot (values empirically determined). Moreover, as fNDs are located inside endosomes, we rejected colocalisation candidates having a fNDs spot size larger than the labelled-endosome one. This led to colocalisations statistics in the $z$-stack volume, but in this volume, only fNDs that were moving at the time of fixation would have been considered for transport parameters measurement. From transport quantification videos, we estimated to $9.0 \pm 0.6 \%$ this fraction of fNDs moving. However, all videos have been recorded at a single focused plane that only displays part of all fNDs spots detected in the whole $z$-stack volume. Considering that fNDs are homogeneously distributed in the sample volume, the single-plane detectable fraction corresponds to the ratio of the depth of field $\left(n \lambda / \mathrm{NA}^{2}=0.42 \mu \mathrm{m}\right.$, for $\left.n=1.33\right)$ over the $1.75 \mu \mathrm{m} z$-stack thickness, which yields $24 \%$. Therefore, assuming that one detects moving fNDs with the same yield in a single focused plane than in the $z$-stack volume (but out-of-focused), we infer a fraction of 
possibly moving fNDs in the analysed $z$-stack volume to be only $24 \% \times 9 \%=2.2 \%$. We applied this correction factor of $2.2 \%$ to $z$-stack raw colocalisation data to obtain the proportion $(\approx 10-40 \%)$ of each type of moving fND-cargoes displayed in Supplementary Fig. 2c.

\section{Microtubule destabilizing-drug experiment.}

Nocodazole powder were purchased from Sigma-Aldrich (St Louis, MO, USA) and resuspended in dimethylsulfoxide (DMSO). Nocodazole was added to the cultures after fND protocol internalization and the DMSO volume added to each well was adjusted to be $0.5 \%$ of the total medium volume per well.

\section{Preparation of oligomeric $A \beta_{1-42}$ peptides.}

$1 \mathrm{mg}$ of synthetic $A \beta_{1-42}$ (Bachem AG, Switzerland) was dissolved in $500 \mu \mathrm{L}$ trifluoroacetic acid (Sigma Aldrich) and sonicated during $10 \mathrm{~min}$. After evaporation under nitrogen, the preparation was processed according to Klein et $a^{34}$. The pattern of oligomers was checked by Western blot (see Fig. 3b), with the following gel composition: 10-20\% TrisTricine, $5 \mu \mathrm{L} A \beta_{1-42}$ preparation at $100 \mu \mathrm{M}$ concentration, and Anti-Human sAPP alpha (clone 2B3) Mouse IgG MoAb antibody (IBL/Tecan AG, Switzerland).

Mark1 and Slc25a12 transgenic mice constructs. We used a 3,200 bp mouse CamKII $\alpha$ and a regulatory sequence of $711 \mathrm{bp}$ intron and polyA SV40 in a pGL3-basic vector. The pGL3-modified vector was realized in two steps: (i) the regulating sequence "intron+polyA SV40" (711 bp) was amplified by PCR from pCDNA1.1 vector with the following primers: forward 5'-TATATCTAGACCCGGGTGGACAAACTACCTACAGAG (XbaI site underlined) and reverse 5'-TATAGGATCCGCGGCCGCAGAAATATGGCGTTGATACC (BamHI site underlined); (ii) the specific promoter "mouse minimal promoter CamKII $\alpha$ " $(3,200 \mathrm{bp})$ was amplified by PCR from mouse brain cDNA with the following primers: forward 5'-TATAGCTAGCGAGATTCTTCCAGCTAGTTC-3' (Nhel site underlined) and reverse 5'-TATAAAGCTTCCGTGCTCCTGAGTGCAAAC-3' (HindIII site underlined). The $\mathrm{XbaI} / \mathrm{BamHI}$ and then the NheI/HindIII digestion products of the amplicon were inserted into the two multiple cloning site of the pGL3 basic vector. E. coli DH5 $\alpha$ cells were cotransformed with these constructs. The coding regions of the mouse Mark1 gene $(2,386 \mathrm{bp})$ and mouse Slc25a12 gene $(2,034 \mathrm{bp})$ were amplified by PCR (sequences of primers and probes are available on request. The two HindIII/SmaI digestion products of the amplicons were inserted into the multiple cloning site of the pGL3 modified vector. $E$. coli DH5 $\alpha$ cells were co-transformed with this shuttle construct. Transgenes were injected in C57/Bl6 fertilized eggs for random integration into the mouse genome (CNRS SEAT/TAAM UPS 44, Villejuif, France).

Mark1 and SIc25a12 DNA extraction \& genotyping. Tissue from mouse hippocampus embryo was digested overnight at $55^{\circ} \mathrm{C}$ in the digesting buffer: Tris $\mathrm{HCl} \mathrm{pH=8}(50 \mathrm{mM})$, EDTA pH=8 $(100 \mathrm{mM}), \mathrm{NaCl}(60 \mathrm{mM})$, SDS $(1 \% \mathrm{w} / \mathrm{v})$, complemented with $15 \mu \mathrm{l}$ of proteinase K (Qiagen N.V., Netherlands). Inactivation was done at $99^{\circ} \mathrm{C}$ for $10 \mathrm{~min}$ and $3 \mu \mathrm{l}$ of $1 \%(\mathrm{w} / \mathrm{v}$ ) RNaseA (Thermo Fisher Scientific, USA) was added prior to incubation at $37^{\circ} \mathrm{C}$ for $90 \mathrm{~min}$. DNA was then extracted in $300 \mu \mathrm{l}$ of Phenol/Chloroform, and after $5 \mathrm{~min}$ centrifugation at $13200 \mathrm{rpm}$, aqueous phase was diluted elsewhere with $10 \%$ of sodium 
acetate ( $3 \mathrm{M}$ concentration). Then, $800 \mu \mathrm{l}$ of cold ethanol were added before freezing the solution at $-80^{\circ} \mathrm{C}$ for $30 \mathrm{~min}$, followed by $30 \mathrm{~min}$ of $13200 \mathrm{rpm}$ centrifugation at $4^{\circ} \mathrm{C}$. Supernatant was removed and the pellet was rinsed at least once with ethanol $70 \%$. The pellet was finally dried at room temperature and resuspended in sterile water. PCR was then performed using primers for Mark1 and Slc25a12 as follow: UPCamKII $\alpha$ GACTAAGTTGTTCGCATCC; DW_Mark1: TGACGTAATGGAGTTTCTACAC; DW_Slc25a12: TTTCAAATGTCACCTCTCCA. Each PCR amplification was prepared by mixing $0.5 \mathrm{U}$ Hifi taq DNA polymerase (Life Technologies, USA) $1 \mu \mathrm{L}$ of $10 \mathrm{x}$ Buffer, $2 \mathrm{mM} \mathrm{MgSO}$, each deoxynucleoside triphosphate at a concentration of $0.2 \mathrm{mM}, 1 \mu \mathrm{M}$ of each of the corresponding primer, and $50 \mathrm{ng}$ of genomic DNA A final volume of $10 \mu \mathrm{L}$ was achieved using water. A negative control using all reagents except template DNA was also included. Reactions were carried out in a Mastercyler ${ }^{\circledR}$ pro $S$ thermocycler (Eppendorf, Germany) using the following cycling conditions: $2 \mathrm{~min}$ at $95^{\circ} \mathrm{C}$ followed by 28 cycles of $95^{\circ} \mathrm{C}$ for $15 \mathrm{sec}, 58^{\circ} \mathrm{C}$ (for Mark1) or $56^{\circ} \mathrm{C}$ (for Slc25a12) for $30 \mathrm{~s}$, and $72^{\circ} \mathrm{C}$ for $30 \mathrm{~s}$. A final extension step at $72^{\circ} \mathrm{C}$ for 7 min was performed after the cycles. After this step, samples were kept at $4^{\circ} \mathrm{C}$ until being electrophoresed in 1.5\% agarose 1xTBE gels. For visualization of electrophoresed PCR products, gels were stained with 1xSYBR® Safe DNA Gel Stain (Life Technologies) and digital images were captured in a InGenius 3 (Syngene, UK).

\section{Mark1 RNA extraction and RT-qPCR.}

Primary neuronal cells were collected in Trizol ${ }^{\circledR}$ (Life Technologies) for RNA extraction as described by the manufacturer. Reverse transcription (RT) was carried out at $50^{\circ} \mathrm{C}$ for 30 min in a $20 \mu \mathrm{l}$ RT mixture containing $1 \mu \mathrm{g}$ total RNA in Maxima First Strand Synthesis kit (Thermo Fisher Scientific) as described by the manufacturer. The cDNAs generated were amplified by real-time PCR, using probes (obtained from Merck Serono, Germany) labeled at their 5' ends with a fluorogenic reporter dye (FAM) and at their 3' ends with a quencher dye (TAMRA). Sequences of primers and probes are available on request. PCR assays had a final reaction volume of $20 \mu \mathrm{l}$, and contained $2 \mathrm{U}$ of Taq polymerase (Applied Biosystems, USA), $10 \mu \mathrm{M}$ primers and fluorogenic probe. PCR was carried out over 40 cycles of $95^{\circ} \mathrm{C}$ for $15 \mathrm{~s}, 60^{\circ} \mathrm{C}$ for $1 \mathrm{~min}$ and $50^{\circ} \mathrm{C}$ for $1 \mathrm{~min}$. We used the DNA Engine Opticon 2 sequence detection system (Bio-rad, USA), with the Opticon Monitor software for data analysis. For each group, the cDNAs synthesized from total RNA were serially diluted to cover the $0.19 \mathrm{ng}$ to $50 \mathrm{ng}$ range for specific mRNAs and $0.98 \mathrm{pg}$ to $250 \mathrm{pg}$ range for $18 \mathrm{~S}$ rRNAs. These serial dilutions were used to construct standard curves for $18 \mathrm{~S}$ and for each gene of interest and to calculate the amounts of RNA. Each qPCR signal was normalized with respect to $18 \mathrm{~S}$ and expressed in arbitrary units.

\section{Mark1 and Slc25a12 proteins extraction and Western Blot.}

Hippocampus were dissected from P15 Mark1 and Slc25a12 transgenic mice and proteins were extracted in $100 \mu \mathrm{l}$ of lysis buffer: Tris $\mathrm{HCl}$ pH7.4 (20 mM), NaCl (100 mM), NP40 $(1 \% \mathrm{v} / \mathrm{v})$ and protease phosphatase inhibitor dose (Thermo Fisher Scientific). After milling and restoring for 30 min on ice, centrifugation was performed at $13200 \mathrm{rpm}$ at $4^{\circ} \mathrm{C}$ for $10 \mathrm{~min}$. Supernatant was then removed and kept at $-80^{\circ} \mathrm{C}$ until Western Blots were performed. Protein electrophoresis migration was performed in 4-20\% Mini-PROTEAN Tetra TGX gels (Bio-Rad, USA) in a TGS buffer (Tris, 5 mM; Glycine, 192 mM; 0.1\% SDS, pH 
8.3) with $40 \mu \mathrm{g}$ of protein lysate after denaturation for $10 \mathrm{~min}$ at $95^{\circ} \mathrm{C}$. Nitrocellulose membranes (Trans-Blot Turbo, Bio-Rad) were used for protein transfer. Membrane was then blocked in TBS $1 \mathrm{X}$ Tween $0.1 \%, 5 \%$ non-fat milk for 1 hour at RT under shaking. Primary antibody Mark1 (ref. 3319S, Cell Signaling, USA) and Slc25a12 (ref. ab107436, Abcam, UK) were incubated overnight at $4{ }^{\circ} \mathrm{C}$ either in $5 \% \mathrm{BSA}$ and in $5 \%$ milk respectively. Normalization was performed using Actin (ref A3854, Sigma Aldrich, USA) staining. Secondary HRP-conjugated antibodies were used in corresponding species and enzyme reaction was activated using Clarity Western ECL Substrate (GE Healthcare, USA), and imaged with ChemiDoc XRS (Bio-Rad). Slc25a12 and Mark1 quantification normalized to Actin were performed using images acquired at the best dynamic range (very few saturated pixels).

\section{Statistical analysis.}

Comparison between two data sets was performed with the statistical analysis tools integrated in Igor Pro (WaveMetrics, USA). The pipeline analysis first applies a Kolmogorov-Smirnoff rank test of the null hypothesis "data follow a Gaussian (normal) distribution". In most cases, normality hypothesis was rejected and thus a Mann-WhitneyWilcoxon comparison two-tailed test was performed. When the normality hypothesis could not be rejected, two-sample two-tailed t-test was performed, after evaluating the null hypothesis of equal variance. Data collection for nocodazole and transgenic mouse experiments were performed blind to the conditions of the experiments. No statistical methods were used to predetermine sample sizes, but our sample sizes are similar to those generally employed in the field ${ }^{20}$. Stars are referred to the following significance level: * for $p<0.05$; ** for $p<0.01$; *** for $p<0.001$.

\section{References}

32. Su, L.-J. et al. Creation of high density ensembles of nitrogen-vacancy centers in nitrogen-rich type Ib nanodiamonds. Nanotechnology 24, 315702 (2013).

33. Chenouard, N., Bloch, I. \& Olivo-Marin, J. C. Multiple hypothesis tracking for cluttered biological image sequences. IEEE Trans. Pattern Anal. Mach. Intell. 35, 2736-2750 (2013).

34. Klein, W. L. A $\beta$ toxicity in Alzheimer's disease: globular oligomers (ADDLs) as new vaccine and drug targets. Neurochem. Int. 41, 345-352 (2002). 


\section{Supplementary Information}

\section{"Fluorescent nanodiamond tracking reveals intraneuronal transport abnormalities induced by brain disease-related genetic risk factors"}

Simon HAZIZA, Nitin MOHAN, Yann LOE-MIE, Aude-Marie LEPAGNOL-BESTEL, Sophie MASSOU, Marie-Pierre ADAM, Xuan Loc LE, Julia VIARD, Christine PLANCON, Rachel DAUDIN, Pascale KOEBEL, Emilie DORARD, Christiane ROSE, Feng-Jen HSIEH, Chih-Che WU, Brigitte POTIER, Yann HERAULT, Carlo SALA, Aiden CORVIN, Bernadette ALLINQUANT, Huan-Cheng CHANG, François TREUSSART and Michel SIMONNEAU

\section{Supplementary Videos}

Supplementary Video 1 | Video from which Fig.1d-e snapshots were extracted. The white frame delimits the neuronal branch in which the endosomes containing fND \#1 or fND \#2 (same as in Fig. 1f kymographs) are moving. The fND locations are indicated during short periods of time by their number. Real time of 20 frames/s.

Supplementary Video 2 | Video of a typical field of view of primary neuron culture after fluorescent nanodiamonds internalization. a, Video at real speed (20 frames/s) showing only the nanodiamonds motion (in red) with some persistence. $\mathbf{b}$, Merge of the video (without persistence) with the bright field DIC image of the same field of view, showing the neuronal branch in which the nanodiamonds move in a directed manner. The yellow star points a nanodiamond that has a fast erratic motion confined around a branch, and which may be bound to a membrane receptor moving back and forth along the branch.

Supplementary Video 3 | Video related to the study of the colocalization of fNDs and lysosomes (labeled with LysoTracker green emitter) recorded by two-colors/dual-view live imaging. The three examples considered correspond to the different situations analyzed in Suppl. Fig. 2. (i) Top: fNDs and lysosomes moving independently (moving fND located outside lysosome); (ii) Middle: one fND inside a lysosome (pointed by the orange arrow) and moving together as evidenced by the yellow color in the merged video on the right; (iii) Bottom: fNDs inside an almost static lysosome (pointed by the pink arrow). Scale bar: $5 \mu \mathrm{m}$; real time, 20 frames/s.

Supplementary Video 4 | Video of fND-containing endosomes having either retrograde or anterograde motion. a, Merged of DIC and TIRF (in red) images, the latter being formed from the accumulation of each TIRF video frame. Most fND traces are connected to a neuron soma located in the upper part of the field of view. b, TIRF video showing four fND-containing endosomes having either a retrograde (fND going up) or anterograde (fND going down) motion in the neuronal branch of a neuron with its soma on further up and not shown (real time, 20 frames/s). The yellow traces left correspond to the trajectories as inferred from our data processing.

Supplementary Video 5 | Video of anterograde and retrograde motions of fNDcontaining endosomes inside the axon of hippocampal neurons cultured in microfluidic chambers. Real time: 20 frames/s. 
Supplementary Video 6 | Video showing one moving QD-containing cargoes (top) and one moving fNDs-containing endosomes (bottom) in neuronal branches of cultured hippocampal neurons. QDs display strong ON-OFF blinking, including the moving one, indicating that they are internalized individually in their cargoes. In contrast fNDs display a very stable fluorescence owing to the perfect photostability of the embedded nitrogenvacancy color centers. The maximum intensity projections are presented at the end of the movie, evidencing more clearly QDs dark periods. Real time: 20 frames/s.

\section{Supplementary Tables}

Supplementary Table 1 | Statistical analysis of intraneuronal transport parameters. All trafficking parameters were extracted from $n=460$ trajectories acquired from $n=11$ C57Bl6 mouse embryos. The range and interquartile range correspond to the $0-100 \%$ and $25-75 \%$ percentiles, respectively.

\begin{tabular}{lccccc} 
Readouts & Range & Median & Mean & $\begin{array}{c}\text { Standard } \\
\text { Deviation }\end{array}$ & $\begin{array}{c}\text { Interquartile } \\
\text { Range }\end{array}$ \\
\hline Velocity $(\mu \mathrm{m} / \mathrm{s})$ & $0.66-3.75$ & 1.31 & 1.45 & 0.54 & $1.07-1.64$ \\
Processivity $(\mathrm{s})$ & $0.14-6.4$ & 0.48 & 0.64 & 0.6 & $0.33-0.72$ \\
Run Length $(\mu \mathrm{m})$ & $0.19-15.54$ & 0.93 & 1.5 & 1.98 & $0.56-1.63$ \\
Pausing Time $(\mathrm{s})$ & $0.05-9.78$ & 0.32 & 0.48 & 1.04 & $0.18-0.53$ \\
Pausing Frequency $(\mathrm{events} / \mathrm{min})$ & $5.2-129.3$ & 64.1 & 64.5 & 24.6 & $48.1-78.5$ \\
Diffusion Coefficient $\left(\mu \mathrm{m}^{2} / \mathrm{s}\right)$ & $0.001-0.071$ & 0.016 & 0.020 & 0.015 & $0.009-0.027$ \\
Signal-to-Noise Ratio & $4.5-61.3$ & 14.8 & 17.1 & 9.5 & $10.1-21.6$
\end{tabular}

Supplementary Table 2 | Comparison between fND-tracking assay and QD-tracking assay, in terms of the nanolabel physical properties, and of the tracking-assay performance index (localization precision, fraction of moving labels, number of trajectories per field of view and number of missed points in trajectory reconstruction). For the localization precision, fNDs and Qdots were deposited on a coverslip and tracked over 100 frames to measure the standard deviation of $(\mathrm{X} ; \mathrm{Y})$ coordinates over time. Because of QDots blinking, we considered the maximum of emission signal to measure the SBR for each particle. Altogether, the table shows that QDs and fNDs have similar physical properties but that the performance indices of fNDs are all more favorable than the ones of QDs. All tracking data have been acquired at the same excitation wavelength $(561 \mathrm{~nm})$ and the same laser power for both nanolabels.

\begin{tabular}{rcc} 
Properties & fND & QD \\
\hline Max emission wavelength & $700 \mathrm{~nm}$ & $705 \mathrm{~nm}$ \\
Mean size & $30 \mathrm{~nm}$ & $25 \mathrm{~nm}$ \\
Surface chemical group & carboxylic functions & carboxylic functions \\
Localisation precision & $15.0 \pm 0.5 \mathrm{~nm}^{\dagger}$ & $24.7 \pm 1.1 \mathrm{~nm}^{\ddagger}$ \\
\# particles / Field of View (FoV) & $65 \pm 3$ & $170 \pm 10$ \\
Moving particles / FoV & $9.1 \pm 0.3 \%$ & $1.4 \pm 0.2 \%$ \\
Missed points / trajectory & $0.8 \pm 0.2 \%$ & $4.4 \pm 0.4 \%$ \\
(cutoff at 10\%) & & \\
\hline
\end{tabular}

+ for a dataset $(n=61)$ with mean $S B R=7.4 \pm 0.2$

$\neq$ for a dataset $(n=58)$ with mean $S B R=7.6 \pm 0.2$ 


\begin{tabular}{|c|c|c|c|c|c|}
\hline & & $\begin{array}{c}\text { fND } \\
\text { (this work) }\end{array}$ & $\begin{array}{c}\text { QD } \\
\text { e.g. Ref.1 }\end{array}$ & $\begin{array}{c}\text { Lipophilic dye } \\
\text { e.g. CM-Dil' }{ }^{2}, \text { LysoTracker }^{3}\end{array}$ & $\begin{array}{c}\text { Fluorescent protein (FP) } \\
\text { e.g. Ref. } 4\end{array}$ \\
\hline \multirow{4}{*}{$\begin{array}{l}\text { Ease of Use } \\
+ \text { Specificity } \\
\text { + Versatility }\end{array}$} & $\begin{array}{l}\text { Labelling } \\
\text { protocol }\end{array}$ & $\begin{array}{l}\text { Simple and fast labelling } \\
\text { protocol by spontaneous } \\
\text { endocytosis of as-produced } \\
\text { nanoparticles. Acquisitions } \\
\text { start only } 30 \text { min after } \\
\text { addition of fNDs; no wash } \\
\text { necessary. }\end{array}$ & $\begin{array}{l}\text { Endocytosis of bio- } \\
\text { conjugated QDs (with Nerve } \\
\text { Growth Factor, NGF) in a sub- } \\
\text { population of endosome. QDs } \\
\text { added } 3 \text { hours before starting } \\
\text { acquisitions; wash necessary. }\end{array}$ & $\begin{array}{l}\text { Long labelling protocol } \\
\text { (2h-4h30 before starting } \\
\text { acquisitions; wash } \\
\text { necessary). } \\
\text { A large fraction of neurons } \\
\text { have labelled vesicles. }\end{array}$ & $\begin{array}{l}\text { Transfection of protein-FP } \\
\text { constructs. Very low yield (a } \\
\text { few \%). } \\
\text { Most studies based on } \\
\text { uncontrolled protein } \\
\text { overproduction that may } \\
\text { cause numerous artifacts } \\
\text { (ectopic sub-cellular } \\
\text { localizations, erroneous } \\
\text { formation of protein } \\
\text { complexes...). } \\
\text { However, mouse knockin } \\
\text { transgenic models are } \\
\text { possible }\end{array}$ \\
\hline & $\begin{array}{l}\text { Type of } \\
\text { cells }\end{array}$ & $\begin{array}{l}\text { fND tracking assay } \\
\text { demonstrated on cultured } \\
\text { hippocampal and cortical } \\
\text { (not presented) neurons. } \\
\text { Both dendritic and axonal } \\
\text { transport studied. }\end{array}$ & $\begin{array}{l}\text { QDs tracking demonstrated } \\
\text { only in Dorsal Root Ganglion } \\
\text { neurons only (due to NGF } \\
\text { receptor enrichment). } \\
\text { Study limited to axonal } \\
\text { transport. }\end{array}$ & All types of neuronal cells. & All types of neuronal cells. \\
\hline & $\begin{array}{l}\text { labelling } \\
\text { specificity }\end{array}$ & $\begin{array}{l}\text { fNDs internalized in all types } \\
\text { of endosomes but almost not } \\
\text { in lysosomes }\end{array}$ & $\begin{array}{l}\text { NGF-QDs labelling specifically } \\
\text { TrkA-vesicles }\end{array}$ & $\begin{array}{l}\text { LysoTracker@: specific of } \\
\text { Lysosomes. } \\
\text { but CM-Dilß labels both } \\
\text { endosomes and lysosomes }\end{array}$ & $\begin{array}{l}\text { Possibility of labelling specific } \\
\text { vesicles with appropriate FP } \\
\text { fusion protein construct. }\end{array}$ \\
\hline & $\begin{array}{l}\text { Other } \\
\text { characte- } \\
\text { ristics }\end{array}$ & $\begin{array}{l}\text { Nitrogen-vacancy }\left(\mathrm{NV}^{-}\right) \\
\text {embedded in fNDs allows } \\
\text { various sensing: } \\
\text { thermometry, } \\
\text { magnetometry }{ }^{6} . .\end{array}$ & - & - & $\begin{array}{l}\text { Possible combination to } \\
\text { Optogenetics } \rightarrow \text { control of } \\
\text { organelle transport and } \\
\text { positioning?. }\end{array}$ \\
\hline \multirow{3}{*}{$\begin{array}{c}\text { Spatio } \\
\text { temporal } \\
\text { resolution } \\
\& \\
\text { Measure- } \\
\text { ment } \\
\text { Sensitivity } \\
\text { and } \\
\text { Reliability }\end{array}$} & $\begin{array}{l}\text { Photo- } \\
\text { physical } \\
\text { Properties }\end{array}$ & $\begin{array}{l}\text { Perfect photostability and } \\
\text { similar brightness as QD. } \\
\text { Inhomogeneous in size and } \\
\text { brightness. } \\
\text { Lack of diversity of emission } \\
\text { wavelengths. }\end{array}$ & $\begin{array}{l}\text { High brightness, good } \\
\text { photostability, but } \\
\text { photoblinking. } \\
\text { Homogeneous size and } \\
\text { brightness. } \\
\text { Broad variety of emission } \\
\text { wavelengths (size tunability). }\end{array}$ & $\begin{array}{l}\text { Bright but not photostable. } \\
\text { Various emission } \\
\text { wavelengths available. }\end{array}$ & $\begin{array}{l}\text { Various emission wavelengths } \\
\text { available. }\end{array}$ \\
\hline & Precisions & $\begin{array}{l}15 \mathrm{~nm} \text { mean localisation } \\
\text { precision at } 20 \text { frames/s } \\
\text { (Suppl. Table 2). }\end{array}$ & $\begin{array}{l}25 \mathrm{~nm} \text { localisation precision } \\
\text { at } 20 \text { frames/s (Suppl. } \\
\text { Table 2). }\end{array}$ & $\begin{array}{l}2 \text { frames/s with CM-Dil, } \\
\text { but } 20 \text { frames/s with } \\
\text { Lysotracker (Suppl. } \\
\text { Video 3). } \\
\text { Spatial resolution } \\
\text { degrading due to } \\
\text { bleaching. }\end{array}$ & $\begin{array}{l}<5 \text { frames/s. } \\
\text { Spatial resolution degrading } \\
\text { due to bleaching. }\end{array}$ \\
\hline & $\begin{array}{l}\text { Transport } \\
\text { parameter } \\
\text { measure- } \\
\text { ments; } \\
\text { Reliability } \\
\quad \text { and } \\
\text { Sensitivity }\end{array}$ & $\begin{array}{l}\text { High throughput and high } \\
\text { content (additional } \\
\text { parameters to velocity and } \\
\text { processivity are extracted) } \\
\text { Reliable measurements } \\
\text { (Suppl. Fig. 8) } \\
\text { Sensitive to slight transport } \\
\text { impairments (Fig. } 3-5 \text { ) }\end{array}$ & $\begin{array}{l}\text { High throughput but low } \\
\text { content: only retrograde } \\
\text { motions and only velocity } \\
\text { and processivity measured. } \\
\text { Not reliable measurements } \\
\text { (Suppl. Fig. 8) }\end{array}$ & $\begin{array}{l}\text { High throughput but } \\
\text { Low content (kymograph- } \\
\text { based analysis). }\end{array}$ & $\begin{array}{l}\text { High throughput but } \\
\text { Low content (kymograph- } \\
\text { based analysis). }\end{array}$ \\
\hline Toxicity & & $\begin{array}{l}\text { No toxicity } \\
\text { Not biodegradable. }\end{array}$ & $\begin{array}{l}\text { Low at short term, depending } \\
\text { on size and surface chemical } \\
\text { function and high toxicity of } \\
\text { the core component }{ }^{9}\end{array}$ & $\begin{array}{l}\text { Low to high depending on } \\
\text { dye }\end{array}$ & $\begin{array}{l}\text { Low to high depending on } \\
\text { proteins and expression } \\
\text { method. }\end{array}$ \\
\hline $\begin{array}{l}\text { Application } \\
\text { for in vivo } \\
\text { recording }\end{array}$ & & $\begin{array}{l}\text { Not demonstrated yet. } \\
\text { Low 2-photon absorption }{ }^{10} \text {. } \\
\text { Possible in vivo imaging } \\
\text { techniques: Time-gated }_{\text {detection }}{ }^{11} \text {, magnetic } \\
\text { modulation of fluorescence }^{12} \text {. }\end{array}$ & $\begin{array}{l}\text { Not demonstrated yet. } \\
\text { Two-photon fluorescence } \\
\text { excitation }{ }^{13} \text { and time-gated } \\
\text { detection possible. }\end{array}$ & Not demonstrated yet. & $\begin{array}{l}\text { In vivo monitoring in various } \\
\text { animals with multiple optical } \\
\text { approaches }{ }^{4,14,15} \text {. } \\
\text { Possible in vivo imaging using } \\
\text { knockin mouse models }{ }^{5} \text {. }\end{array}$ \\
\hline
\end{tabular}

Supplementary Table 3 | Comparison of the fND-tracking assay with published methodologies used to monitor the intraneuronal transport, regarding ease-of-use, spatio-temporal resolution, toxicity and potential in vivo applications. In particular, we compared our approach with the one using QDs, first reported in Cui et al ${ }^{1}$, and the one of Nakamura et. al. ${ }^{2}$ using lipophilic dye CM-DiI. We also included the fluorescent protein category as it is a well-established and versatile molecular cell biology technique. Footnote citations refer to the list of references provided in Supplementary Text 1. Red and green colors indicate pros and cons properties of each technique. Orange color codes 
for what we consider as a moderate drawback in the context of intraneuronal transport measurement.

\section{Supplementary Text 1 | List of references cited in Supplementary Table 3}

1. Cui, B. et al. One at a time, live tracking of NGF axonal transport using quantum dots. Proc. Natl. Acad. Sci. U. S. A. 104, 13666-71 (2007).

2. Nakamura, H. et al. Quantitative analysis of intraneuronal transport in human iPS neurons. J. Pharmacol. Sci. 128, 170-178 (2015).

3. Ori-McKenney, K. M., Xu, J., Gross, S. P. \& Vallee, R. B. A cytoplasmic dynein tail mutation impairs motor processivity. Nat. Cell Biol. 12, 1228-1234 (2010).

4. Yau, K. W. et al. Dendrites In Vitro and In Vivo Contain Microtubules of Opposite Polarity and Axon Formation Correlates with Uniform Plus-End-Out Microtubule Orientation. J. Neurosci. 36, 1071-1085 (2016).

5. Park, H. Y. et al. Visualization of Dynamics of Single Endogenous mRNA Labeled in Live Mouse. Science. 343, 422-424 (2014).

6. van Bergeijk, P., Adrian, M., Hoogenraad, C. C. \& Kapitein, L. C. Optogenetic control of organelle transport and positioning. Nature 518, 111-114 (2015).

7. Schirhagl, R., Chang, K., Loretz, M. \& Degen, C. L. Nitrogen-Vacancy Centers in Diamond: Nanoscale Sensors for Physics and Biology. Annu. Rev. Phys. Chem. 65, 83-105 (2014).

8. Huang, Y.-A. et al. The effect of fluorescent nanodiamonds on neuronal survival and morphogenesis. Sci. Rep. 4, 6919 (2014).

9. Oh, E. et al. Meta-analysis of cellular toxicity for cadmium-containing quantum dots. Nat. Nanotechnol. 11, 479-486 (2016).

10. Wee, T.-L. et al. Two-photon excited fluorescence of nitrogen-vacancy centers in proton-irradiated type Ib diamond. J. Phys. Chem. A 111, 9379-86 (2007).

11. Hui, Y. Y. et al. Wide-field imaging and flow cytometric analysis of cancer cells in blood by fluorescent nanodiamond labeling and time gating. Sci. Rep. 4, 5574 (2014).

12. Sarkar, S. K. et al. Wide-field in vivo background free imaging by selective magnetic modulation of nanodiamond fluorescence. Biomed. Opt. Express 5, 1190202 (2014).

13. Biermann, B. et al. Imaging of molecular surface dynamics in brain slices using single-particle tracking. Nat. Commun. 5, 3024 (2014).

14. Zala, D. et al. Vesicular glycolysis provides on-board energy for fast axonal transport. Cell 152, 479-491 (2013).

15. Drerup, C. M. \& Nechiporuk, A. V. In vivo analysis of axonal transport in zebrafish. Methods Cell Biol. 131, (2015). 


\section{Supplementary Figures}
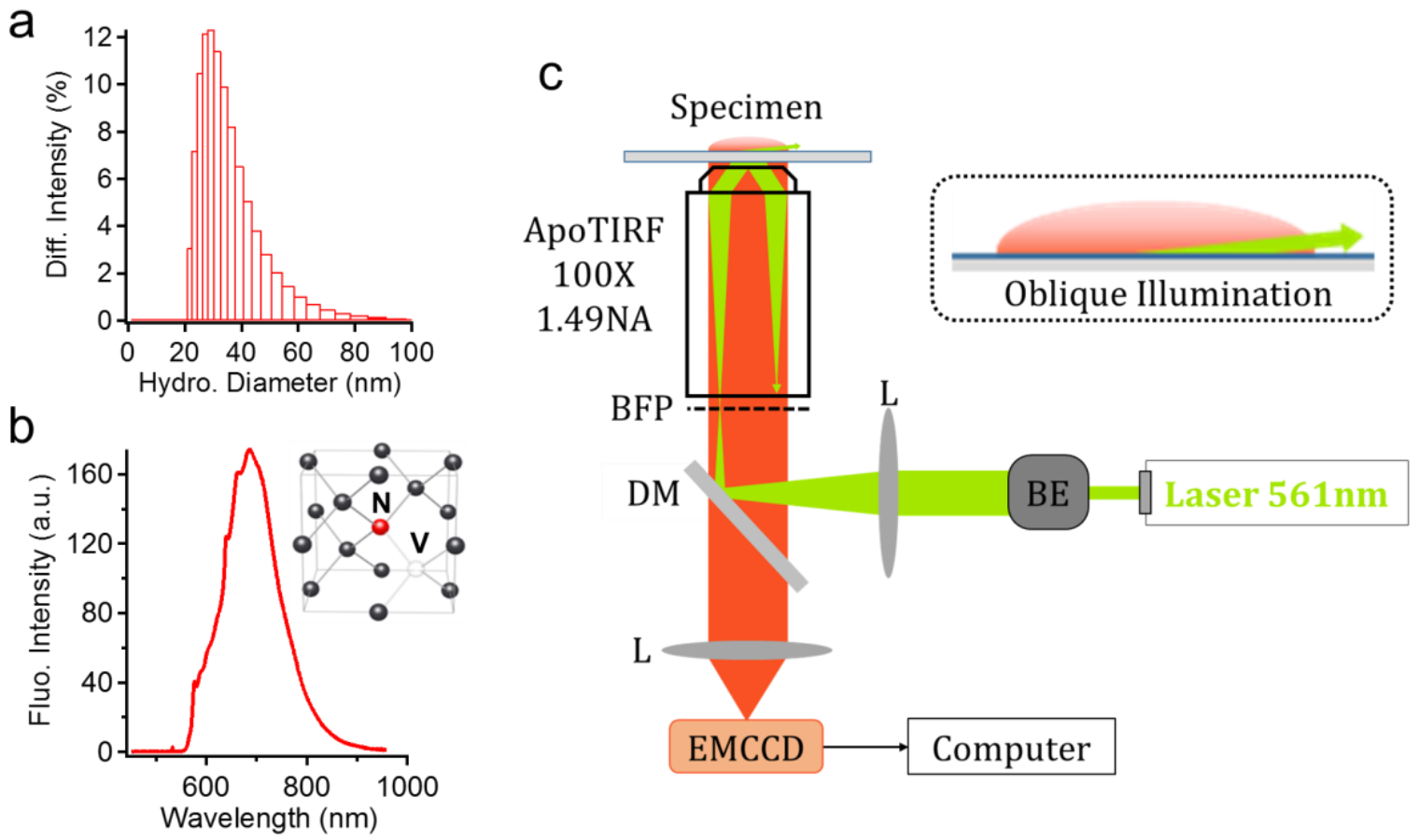

Supplementary Figure 1 | fNDs properties and video-microscopy set-up. a, Size distribution of the fND solution as measured by dynamic light scattering. fND mean size is $30 \mathrm{~nm}$. b. Photoluminescence spectrum of a single fND, excited with a laser at $561 \mathrm{~nm}$ wavelength. Inset: $\mathrm{NV}$ point defect in the diamond crystallographic lattice. c, Home-made total internal reflection fluorescence video-microscope, providing oblique illumination. BE: beam expander, L: lenses, DM: dichroic beamsplitter, BFP: back focal plane, ApoTIRF: apochromatic oil immersion microscope objective of $100 \mathrm{x}$ magnification and 1.49 numerical aperture, EMCCD: electron-multiplying charged-couple device array detector. To study the colocalization of fNDs with endosomal compartments (Suppl. Fig. 2) we added the DualView beam splitter (see Methods) in front of the EMCCD. 


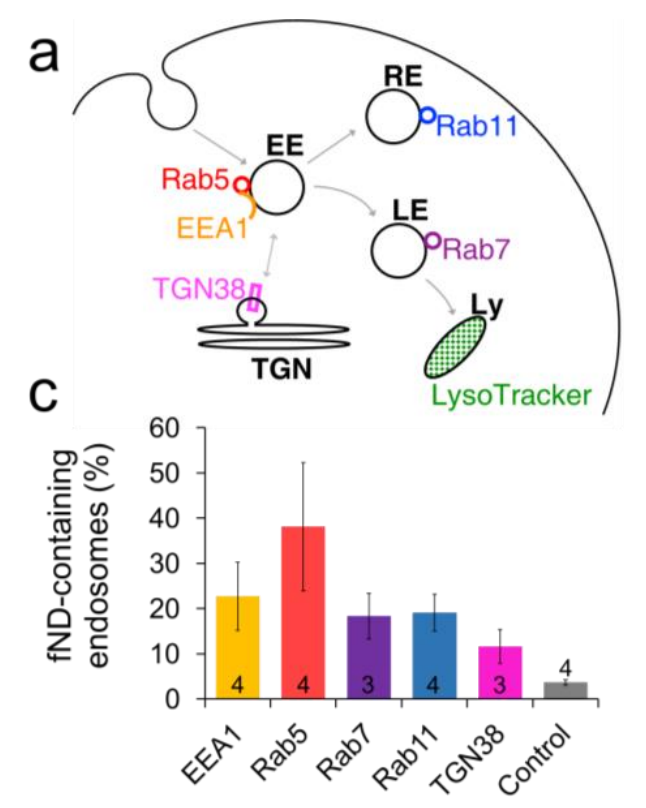

b
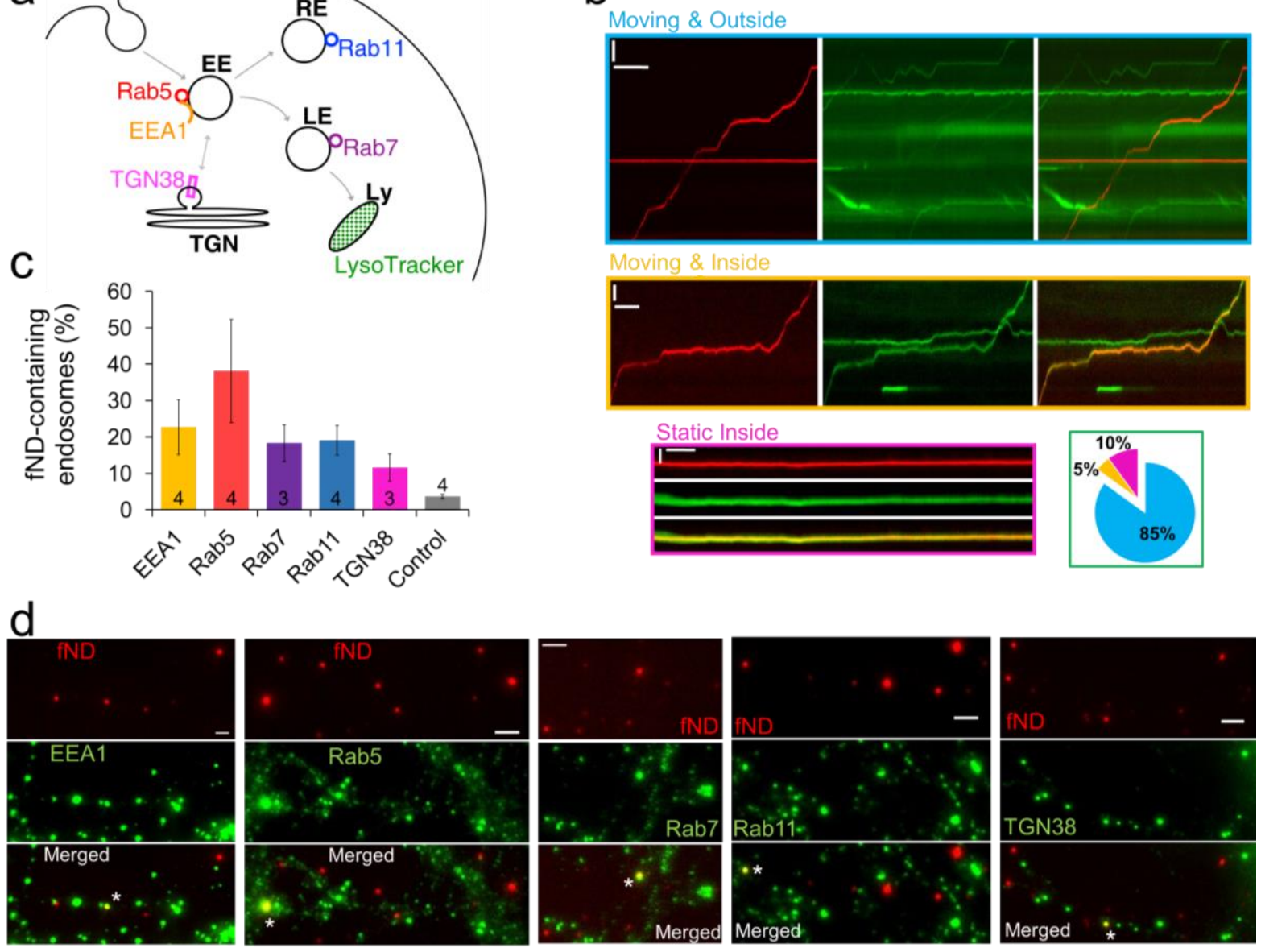

Supplementary Figure 2 | Identification of the fND-containing cargoes. a, Scheme showing the endosome and lysosome possible cargoes at different stages of their development, each characterized by specific membrane protein markers: EEA1 and Rab5 for Early Endosome (EE); Rab7 for Late Endosome (LE); Rab11 for Recycling Endosome (RE); LysoTracker for the Lysosome (Ly) and TGN38 for the Trans Golgi Network to which EE can partly fuse. b, Colocalization with Lysosomes (see also Suppl. Movie 3): Kymographs from simultaneous dual-color live neuron imaging with fND at the left (in red), Lysosomes in the middle (green), and the merged image at the right. Three cases are presented for $n=100$ internalized fNDs studied (either moving or having a static colocalization with a lysosome): (i) moving fND being outside a lysosome (top kymographs, horizontal \& vertical scale bars of $5 \mathrm{~s}$ and $5 \mu \mathrm{m}$ respectively), representing $85 \%$ of internalized fNDs; (ii) moving fND being inside a lysosome as evidenced by the dynamical colocalization with a moving lysosome (middle), which corresponds to $5 \%$ of internalized fNDs (scale bars: $2 \mathrm{~s}$ and $2 \mu \mathrm{m}$ ); and static fND colocalizing with static lysosomes (bottom, scale bars: $3 \mathrm{~s}$ and $2 \mu \mathrm{m}$ ), representing $10 \%$ of internalized fNDs. c, Colocalization with endosomes: Fractions of fNDs colocalizing with one of the endosomal markers detected by immunofluorescence (see Methods). The "control" bar corresponds to colocalizations of fNDs with non-specific secondary antibodies Alexa488 (no primary antibody). Data displayed have been corrected (multiplicative factor) to take into account the fraction (2.2\%) of moving fNDs only (see Methods). Note that the sum of all contributions is $\approx 110 \%$. However, as EEA1 is an effector of Rab5, it is always associated to this marker, and its contribution has to be excluded from the total, which in turn is 
$\approx 90 \%$. d, Examples of colocalizations (shown by white stars in merged images) between fND and the different endosomal markers. Scale bars: $2 \mu \mathrm{m}$.
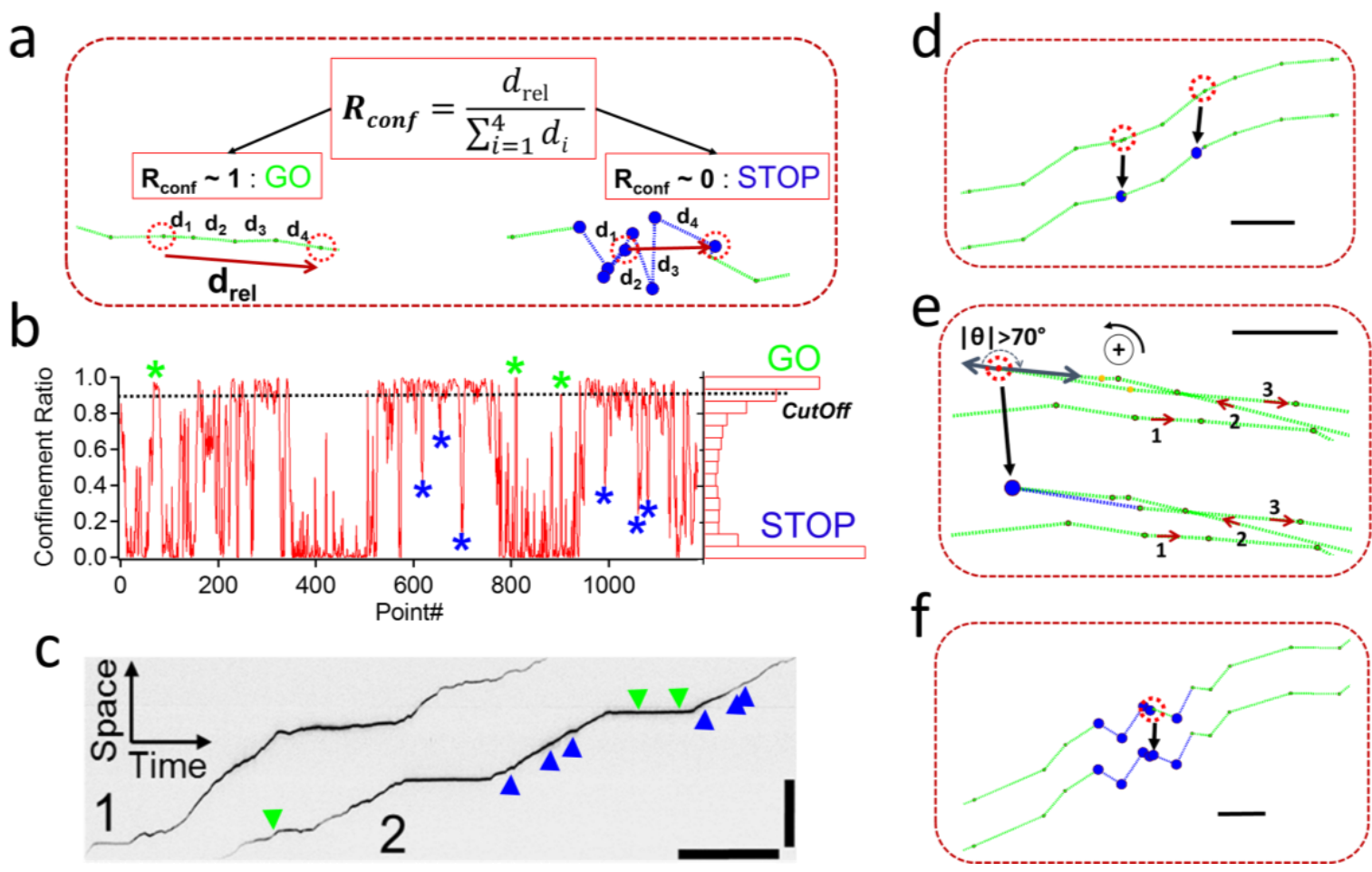

Supplementary Figure 3 | Detection of the "stop-and-go" motion phases. a-b, Coarse detection of "go" phases. a, The confinement ratio function $\mathrm{R}_{\text {conf }}$ is calculated for each trajectory point, using a forward sliding window of four consecutive points, as the relative distance $d_{\text {rel }}$ between extreme positions $i=1$ and $i=4$ over the absolute distance $\Sigma d_{i}$

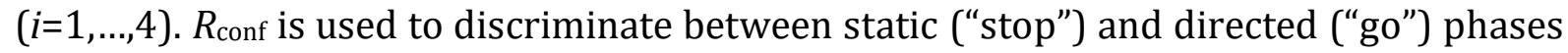
of motion, where $R_{\text {conf }} \approx 1$ corresponds to an endosome in a directed motion "go" state, and $R_{\text {conf }} \approx 0$ corresponds to the "stop" state. $\mathbf{b}$, Graphic representation of Rconf function applied to trajectory \#2 (Fig. 1), revealing the presence of these two phases, with a

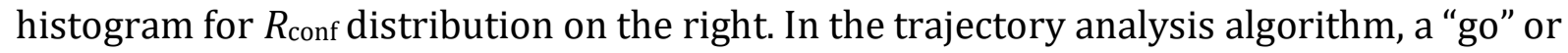
"stop" state is assigned to each trajectory point as a function of its $R$ conf value relative to a cutoff fixed at $R_{\text {conf }}{ }^{\text {cutoff }}=0.9$ (horizontal dashed black line). Green and blue stars referred to short GO and STOP phase respectively. c, Kymograph of trajectory\# 1 and \#2 showing green and blue arrow heads pointing out the same position as stars in (b). These events are usually missing when kymograph is used to infer velocity, run length and pausing time, leading to erroneous measurements. (d-f) Refined detection of "stop" phases. d, If two consecutive "go" state positions (depicted in green) are separated by a distance too small to be determined with precision (below the resolution of this method), the first is considered to be a "stop". Scale bar: $100 \mathrm{~nm}$. e, If the angle between two consecutive segments of motion is larger than 70 degrees, the point separating the two segments is considered to correspond to a "stop". Scale bar: $50 \mathrm{~nm}$. f, If a point within a "stop" region but is identified as a "go", it is considered to be a positive false and is reassigned as a "stop". Scale bar: $100 \mathrm{~nm}$. 

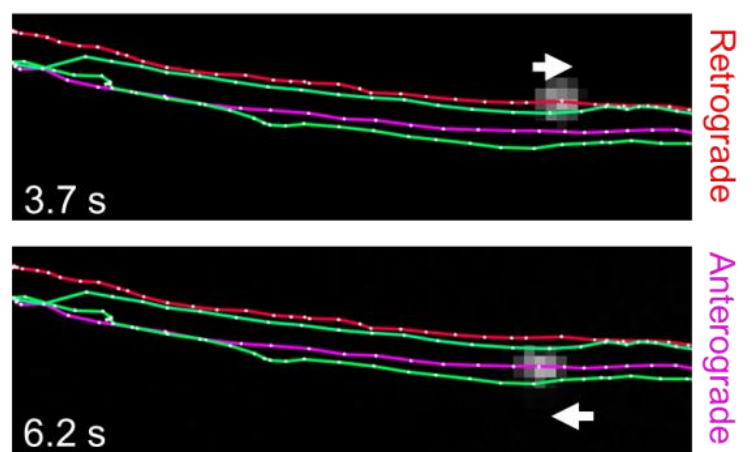

$6.2 \mathrm{~s}$
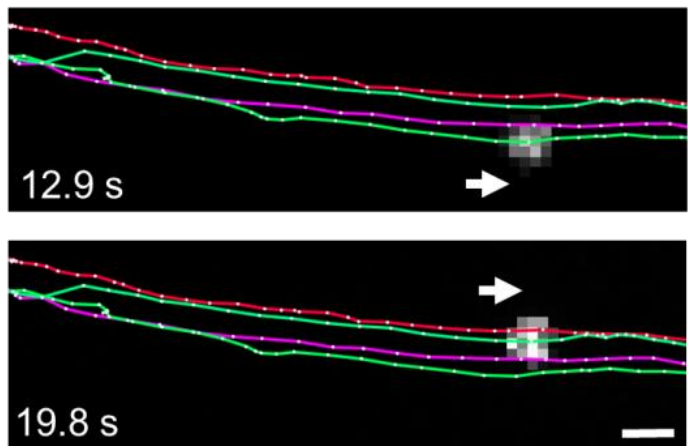

Supplementary Figure 4 | Superresolution mapping of a microtubule bundle by multiple fND tracking. Four trajectories of fND-containing endosomes, revealing underlying well separated microtubules within the same neuronal branch (see Supplementary Video 4). The endosomes are driven by kinesin (left, trajectories in red and purple colors) or dynein (right, trajectories in green and turquoise colors) motors. Scale bar: $400 \mathrm{~nm}$. 
a
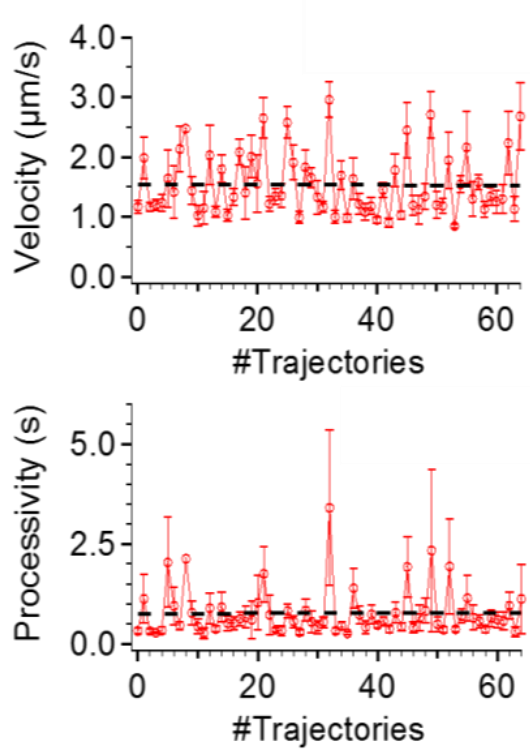
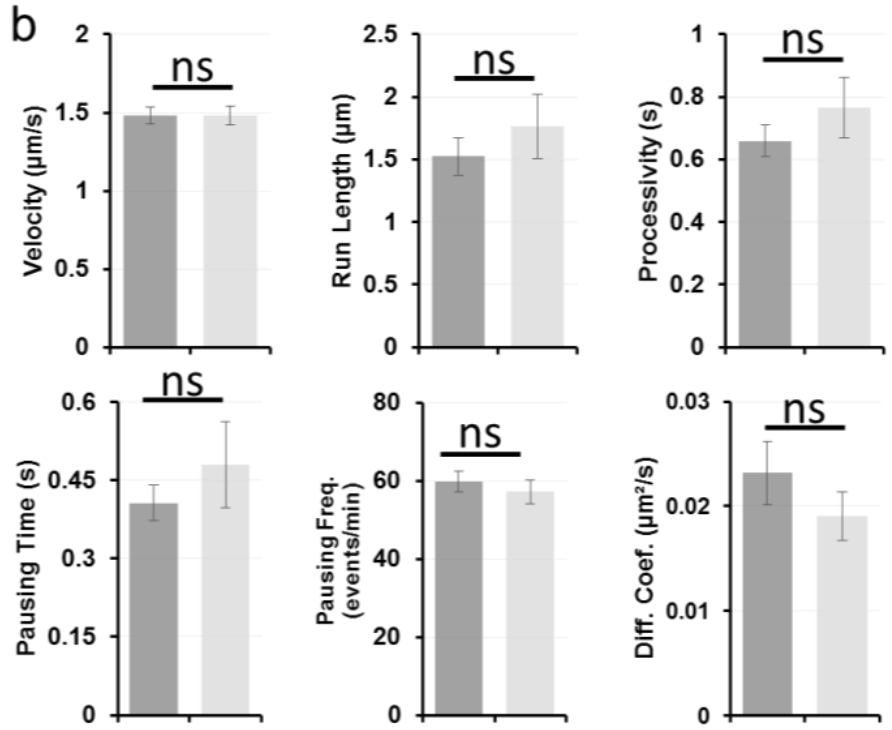

First $15 \mathrm{~min}$

Last $15 \mathrm{~min}$

Supplementary Figure 5 | Intra-experiment stability of the method. a, Variation of two readouts (mean values of velocity and processivity) for 64 successive trajectories acquired from a single hippocampal neuron culture over a total duration of 45 minutes. The dashed black lines are linear fits to the data. Their slopes are $-3.9 \times 10^{-4}$ and $5.7 \times 10^{-4}$ respectively, very close to 0 , indicating a lack of dependence of the experimental procedure on time. $\mathbf{b}$, Comparison of the intraneuronal transport readouts between the first 15 minutes and the last 15 minutes of acquisition, for experiments carried out in triplicate. No statistically significant changes are observed. c, Corresponding Wilcoxon Utest $p$-values.
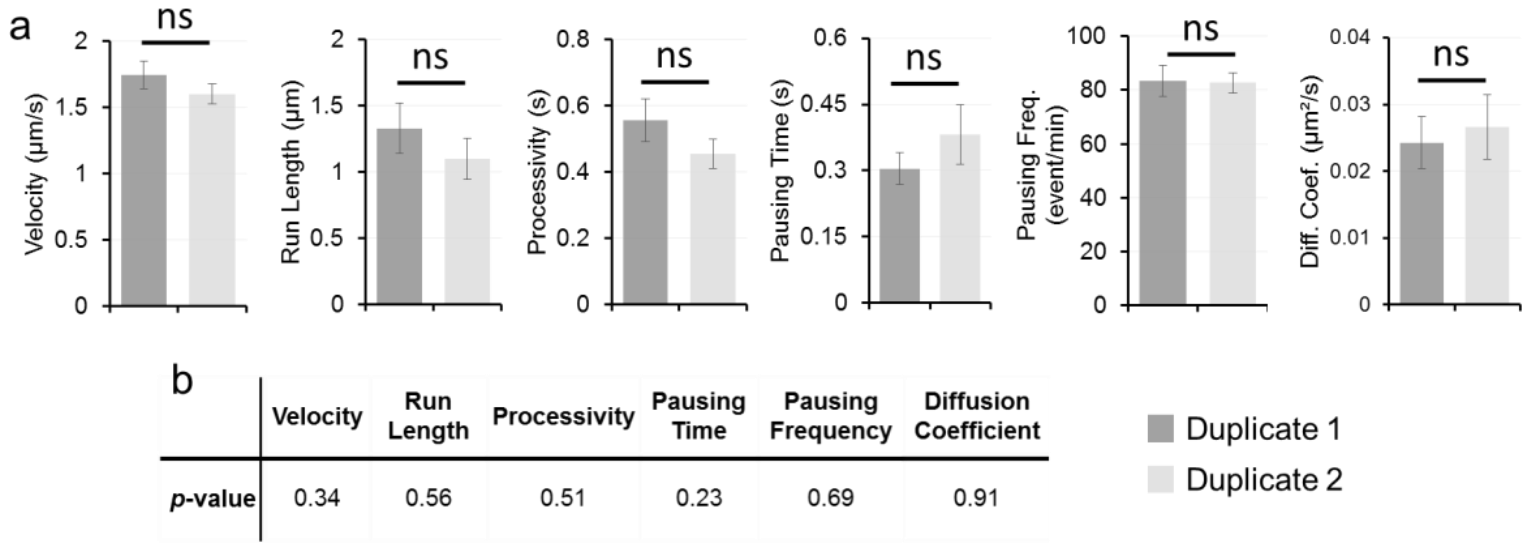

Duplicate 1

Duplicate 2

Supplementary Figure 6 | Inter-experiment stability of the method (technical and biological replicates). a, Intraneuronal transport parameters for two different experiments: two cultures (DIV 11) and two different pregnant females ( $n>30$ trajectories from $n=4$ embryos each experiment). b, Corresponding Wilcoxon U-test $p$-values. 

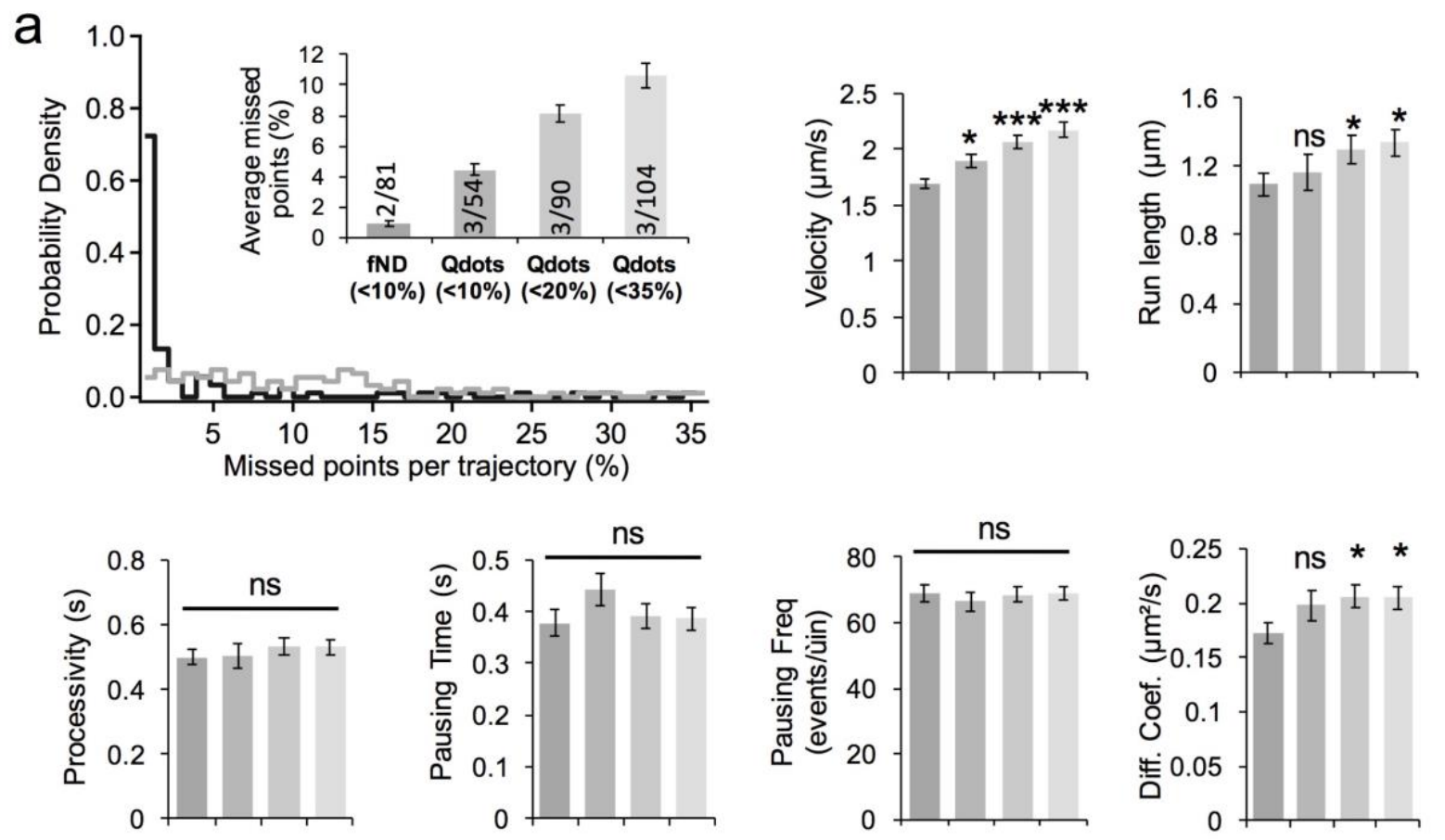

\begin{tabular}{c|cccccc}
\multicolumn{1}{c}{$\boldsymbol{p}$-value } & Velocity & Run Length & Processivity & $\begin{array}{c}\text { Pausing } \\
\text { Time }\end{array}$ & $\begin{array}{c}\text { Pausing } \\
\text { Frequency }\end{array}$ & $\begin{array}{c}\text { Diffusion } \\
\text { Coefficient }\end{array}$ \\
\hline fND / QD 10\% & 0.013 & 0.82 & 0.33 & 0.06 & 0.47 & 0.17 \\
fND / QD 20\% & $10^{-7}$ & 0.045 & 0.65 & 0.61 & 0.76 & 0.023 \\
fND / QD 35\% & $10^{-8}$ & 0.021 & 0.57 & 0.75 & 0.79 & 0.032
\end{tabular}

b
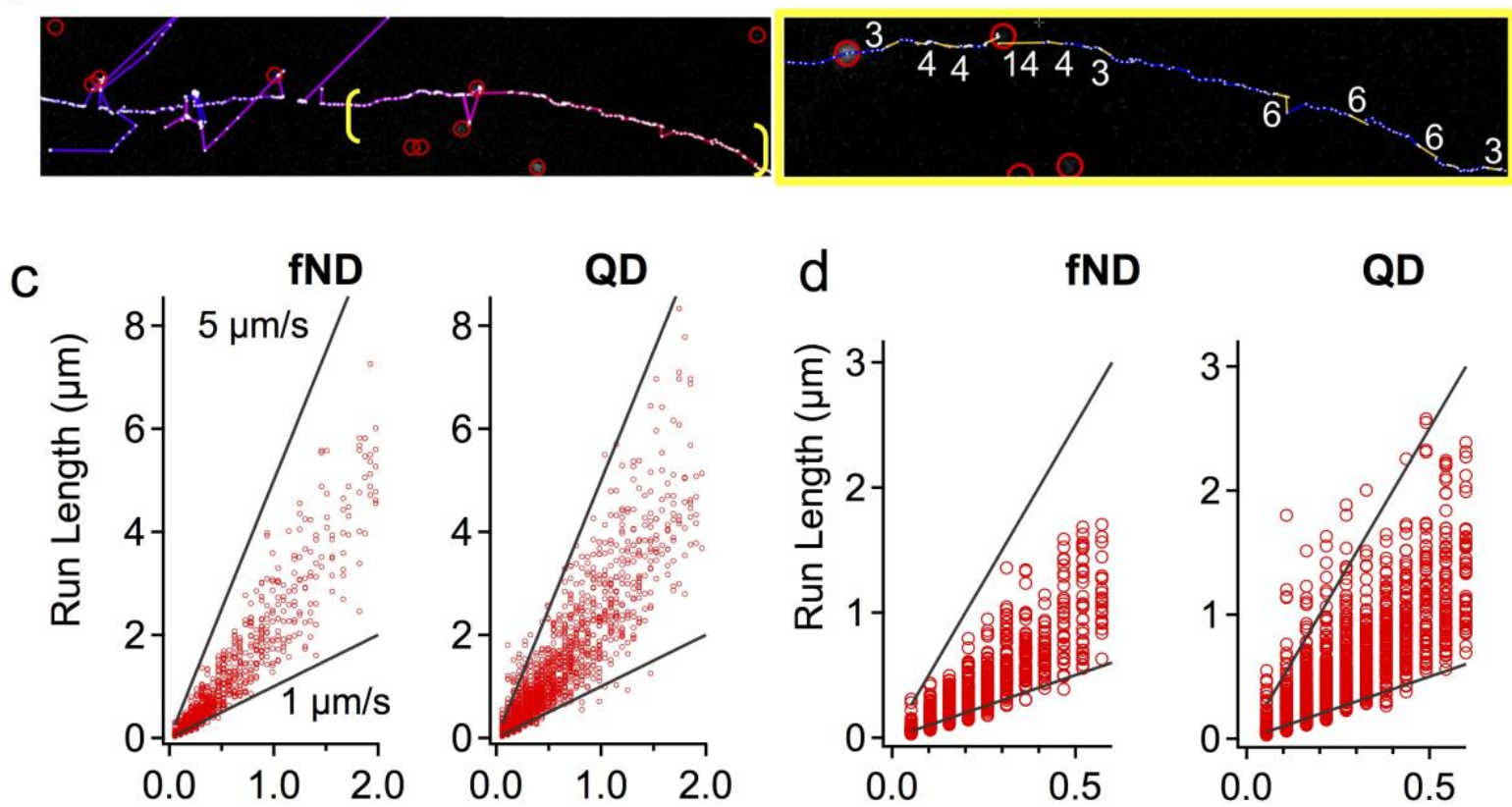

d

fND

QD

Processivity (s) Processivity (s)

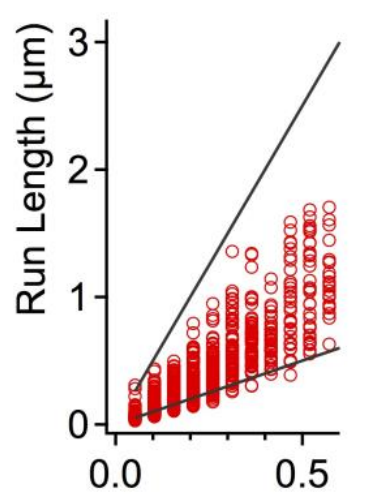

Processivity (s)

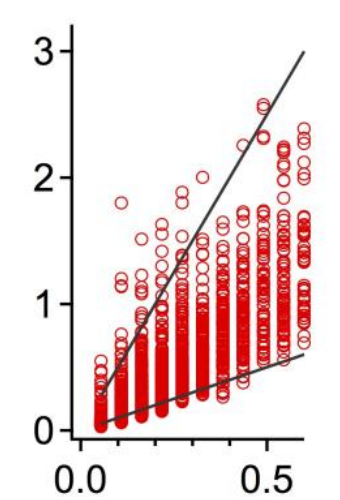

Processivity (s)

Supplementary Figure 7 (caption in the next page) 
Supplementary Figure 7 | Failure of QDs to provide reliable transport parameters. a, Distributions of missed points (absence of nanoparticle signal either due to defocus and due to blinking in the case of QDs) per trajectory in tracking analysis of QDs (grey) and fNDs (black) labeled cargoes. For QDs, the missed-point fraction is mostly homogeneously distributed in a broad range (0-35\%) while it is peaked around zero for fNDs. The histogram in inset shows the average fraction of missed detections for a given dataset. The trajectories from the three QD datasets were selected based on a maximum fraction of missed points/trajectory accepted (low-pass filtering with 10, 20 and 35\% cutoffs). Vertical figures in the bars correspond to: \# of experiments/\# of filtered trajectories. To get a larger number of filtered trajectories from QDs analysis than from fNDs at $10 \%$ cutoff, the QDs missed-points/trajectory cutoff has to be set at 20\%. Note that for similar size of datasets, 3 experiments need to be conducted for QDs against only 2 for fNDs, due to a lower internalization efficiency of QDs, as summarized in Suppl. Table 2. Moreover, the fNDs $10 \%$ cutoff corresponds to an average of only $\approx 1 \%$ missed points/trajectory (cargoes going out of focus), while the $20 \%$ cutoff for QDs is associated to $\approx 8 \%$ missed points/trajectory due to blinking. When performing the transport parameter calculation from the filtered trajectories, we observed that space-dependent parameters (velocity, run length and diffusion coefficient) increased with increasing missed-point fraction cutoff, and differed from values inferred from fND-tracking (considered as ground truths). This can be interpreted as the direct consequence of ICY Spot Tracker algorithm which infers missed points (QD dark period) and creates aberration. Bottom: $p$-values from Wilcoxon U-test. fND/QDs $m m \%$ corresponds to the comparison of fND to QD data for different missed-point fractions $(m m=10-35 \%)$.

b, Left: Portion of a QD trajectory (purple lines) as determined with ICY Spot Tracker. The red circles indicate QDs spots as detected by ICY Spot Detector plugin in a single frame. In the left part of the trajectory, where the QD has long dark periods, the tracking algorithm is not able to connect spots from the moving QD together and therefore create aberrant segments. In the opposite, the right portion in yellow brackets shows full connectivity. Right: despite the continuity, a significant fraction of the trajectories is made of inferred segments (in yellow; white numbers are in pixel unit). These aberrant segments are only one time-frame long (50 ms) and can be as large as 14 pixels (i.e. $\approx 1.1 \mu \mathrm{m}$ ), which correspond to an aberrant velocity of $22 \mu \mathrm{m} / \mathrm{s}$.

c) Comparison of scattered plot of run length vs processivity for fNDs (left) and QDs (right). Black lines indicate extremal cargoes velocities $(1 \mu \mathrm{m} / \mathrm{s}$ and $5 \mu \mathrm{m} / \mathrm{s}$ lower and upper bounds respectively) as reported in the literature (e.g. D. Zala et al., Cell 152, 479 (2013)) and consistent with fNDs measurements. The QD scattered plot shows a significant fraction of velocities larger than the upper bound, especially at short processivities (as displayed in the zoom of panel d). Therefore, the more we increase the fraction of missed point per trajectory (from $10 \%$ to $35 \%$ ), the more we increase the proportion of such aberrant spatial displacements. This will thus affect upward the values of the run length, the velocity and the diffusion coefficient, as observed in a. Altogether, these results demonstrate that QDs cannot be used to measure the intraneuronal transport parameters as it will lead to biologically irrelevant values. 
a

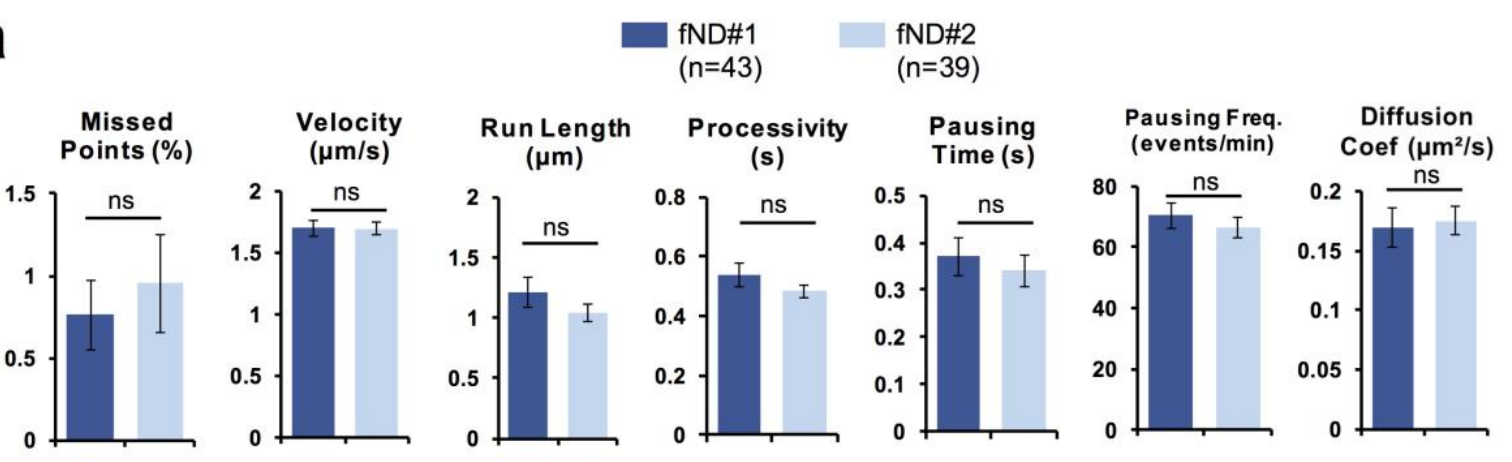

b
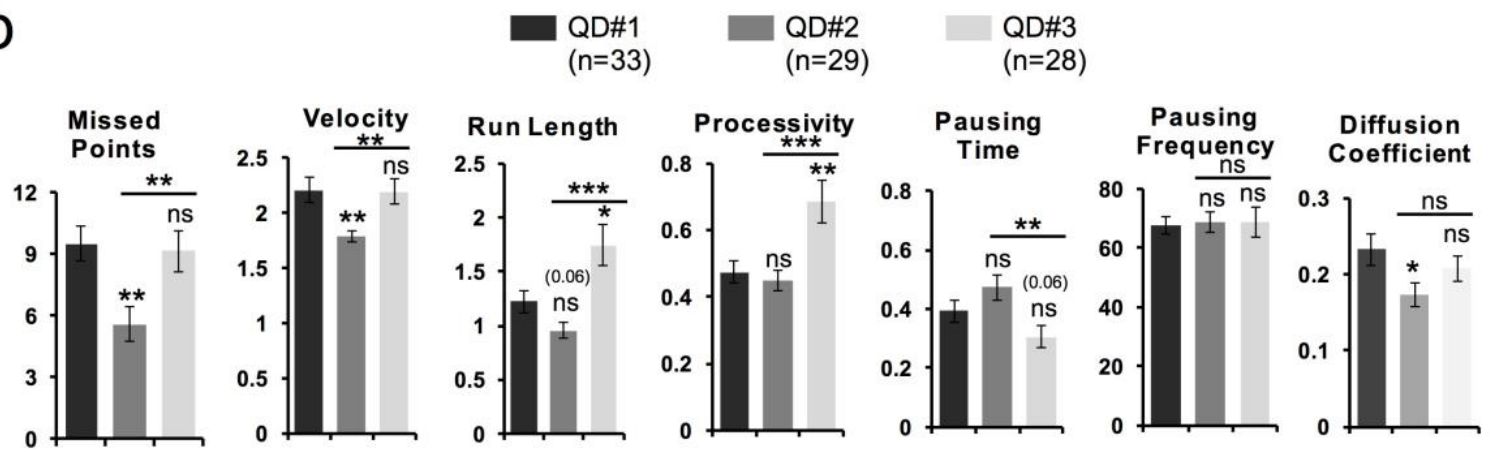

\begin{tabular}{|c|c|c|c|c|c|c|}
\hline$p$-value & Velocity & $\begin{array}{l}\text { Run } \\
\text { Length }\end{array}$ & Processivity & $\begin{array}{c}\text { Pausing } \\
\text { Time }\end{array}$ & $\begin{array}{l}\text { Pausing } \\
\text { Frequency }\end{array}$ & $\begin{array}{l}\text { Diffusion } \\
\text { Coefficient }\end{array}$ \\
\hline fND\#1 / fND\#2 & 0.63 & 0.8 & 0.94 & 0.71 & 0.76 & 0.27 \\
\hline QD\#1 / QD\#2 & 0.002 & 0.06 & 0.6 & 0.14 & 0.96 & 0.03 \\
\hline QD\#1 / QD\#3 & 0.99 & 0.03 & 0.002 & 0.06 & 0.72 & 0.4 \\
\hline QD\#2 / QD\#3 & 0.005 & 0.0001 & 0.0003 & 0.003 & 0.86 & 0.11 \\
\hline
\end{tabular}

Supplementary Figure 8 | Comparison of the inter-experiment stability of QD and fND tracking assays (technical replicates). a, Average missed points/trajectory and intraneuronal transport parameters as inferred from fNDs tracking for two different experiments (fND\#1 and \#2) corresponding to two different coverslips from the same neuron culture (at DIV 15), contrary to Suppl. Fig. 5 (two different cultures). The number $n$ of trajectories taken into account is indicated in bracket. b, Average missed points/trajectory and intraneuronal transport parameters as inferred from QDs tracking for three different experiments (QD\#1, \#2 and \#3), corresponding to three different coverslips from the same neuron culture (at DIV 15). We observe that most transport parameters differ from one coverslip to the other, indicating that we cannot apply our nanoparticle-tracking assay with QDs for intraneuronal transport measurements. c, Corresponding Mann-Whitney-Wilcoxon test $p$-values. 

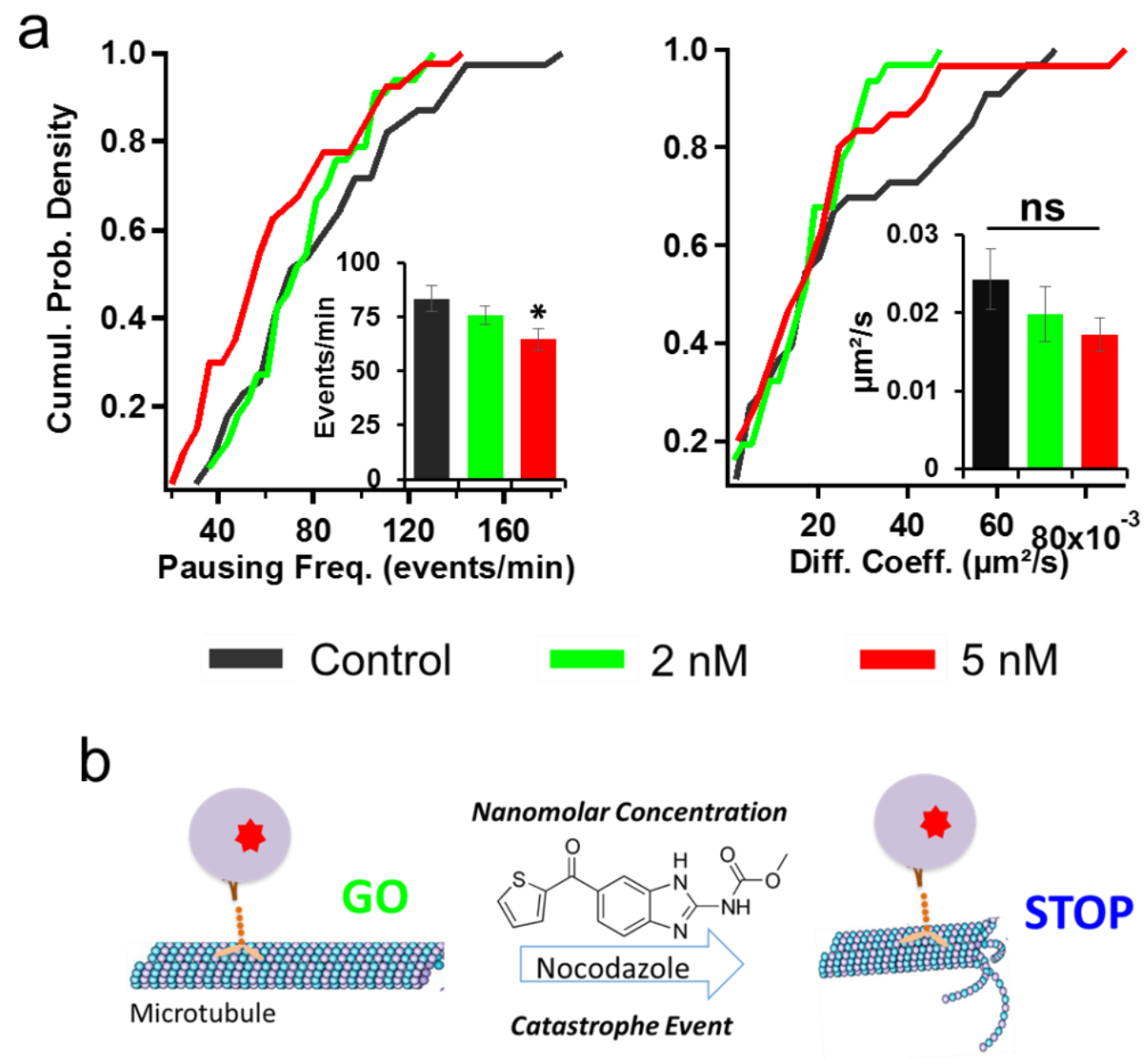

Supplementary Figure 9 | Additional intraneuronal transport parameters for nocodazole-mediated MT depolymerization. a, Cumulative probability densities with bar plots in the inset (mean \pm SEM) for two readouts for the control (black), $2 \mathrm{nM}$ (green) and $5 \mathrm{nM}$ (red) nocodazole ( $n>35$ trajectories from $n=4$ embryos). Left: Pausing frequency decreases from $83.5 \pm 5.8$ events/min to $75.8 \pm 4.4$ events $/ \mathrm{s}$ and $64.7 \pm 5.0$ events $/ \mathrm{min}$ ( $p=0.54, p=0.016$ respectively). Right: the diffusion coefficient was not significantly affected by the addition of nocodazole ( $p$-values of $p=0.989, p=0.479, p=0.575$, respectively, for $2 \mathrm{nM}$ nocodazole, and $p=0.379, p=0.18, p=0.544$, respectively, for $5 \mathrm{nM}$ nocodazole). $\mathbf{b}$, Schematics of the depolymerization action of nocodazole at the +end microtubule tip, inducing a change of motion state of the fND-containing endosome from a "go" phase to a "stop" state. 

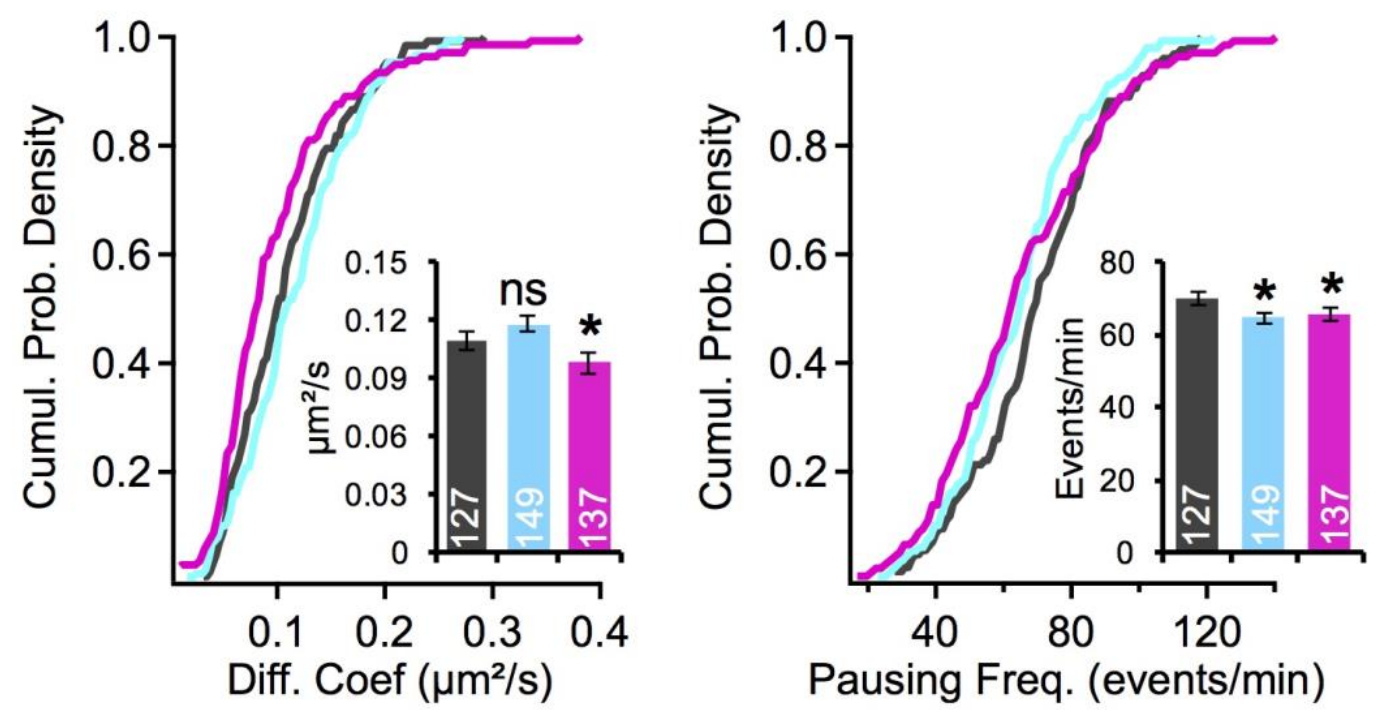

\begin{tabular}{c|cccccc}
$p$-value & Velocity & $\begin{array}{c}\text { Run } \\
\text { Length }\end{array}$ & Processivity & $\begin{array}{c}\text { Pausing } \\
\text { Time }\end{array}$ & $\begin{array}{c}\text { Pausing } \\
\text { Frequency }\end{array}$ & $\begin{array}{c}\text { Diffusion } \\
\text { Coefficient }\end{array}$ \\
\hline Ctrl/0.5 $\mu \mathrm{M}$ & 0.86 & 0.66 & 0.22 & 0.0014 & 0.017 & 0.15 \\
Ctrl/1 $\mu \mathrm{M}$ & 0.015 & 0.0003 & 0.0004 & 0.002 & 0.043 & 0.013
\end{tabular}

Supplementary Figure 10 | Additional intraneuronal transport parameters for Alzheimer Disease-related A $\boldsymbol{\beta}_{1-42}$. Cumulative probability densities with bar plots in the inset (mean \pm s.e.m.) for diffusion coefficient (left) and pausing frequency (right). Control (black), $A \beta_{1-42} 0.5 \mu \mathrm{M}$ concentration (blue) and $A \beta_{1-42} 1 \mu \mathrm{M}$ (purple). Data pooled from $n=2$ experiments each. The number of trajectories is indicated in the bars. Bottom : table of $p$-value for all transport readouts. 

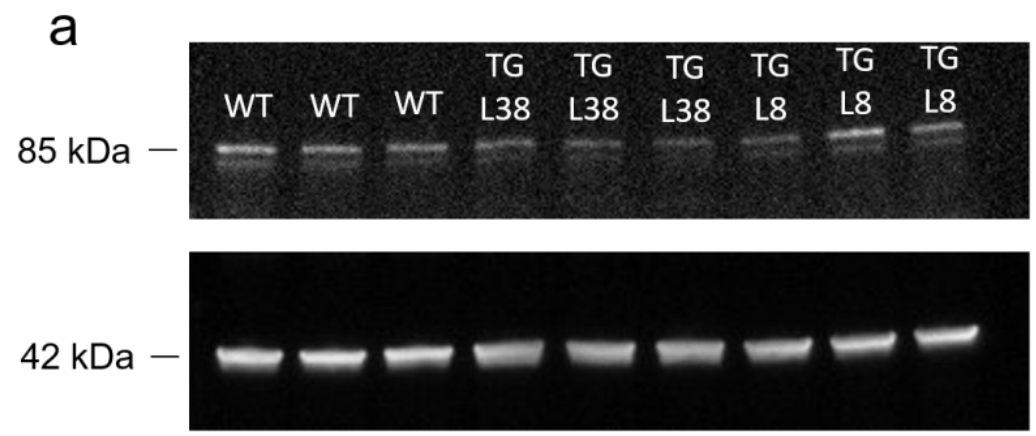

b

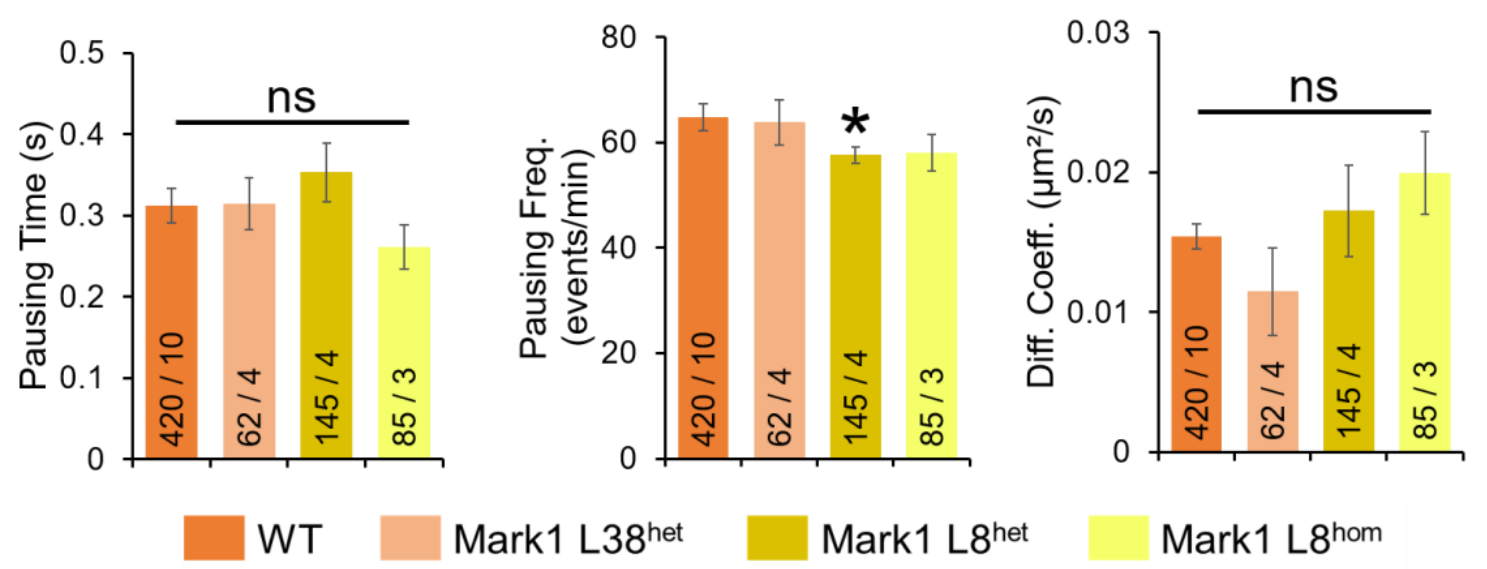

Supplementary Figure 11 | Quantification of the overexpression of Mark1 in the hippocampus in transgenic lines and additional intraneuronal transport parameters. a-b, Western blotting (WB) was performed on hippocampal tissue dissected from P15 mice of the Mark1-transgenic lines L $8^{\text {het }}$ and L38 ${ }^{\text {het, }}$, with comparison to WT mice. WB was carried out with an antibody against Mark1 (a) and an antibody against actin (b), ( $n=3$ P15 mice shown, n=10 performed). b, Pausing time, pausing frequency, and diffusion coefficient displayed no (or very little) significant differences for the Mark1transgenic lines L38 het, $\mathrm{L} 8^{\text {het }}$ and L $8^{\text {hom }}(p=0.684, p=0.787, p=0.164$, respectively, for L38 ${ }^{\text {het; }} p=0.413, p=0.044, p=0.337$, respectively, for L8 ${ }^{\text {het; }} p=0.252, p=0.252, p=0.187$, respectively, for $\mathrm{L} 8^{\mathrm{hom}}$ ). 
a
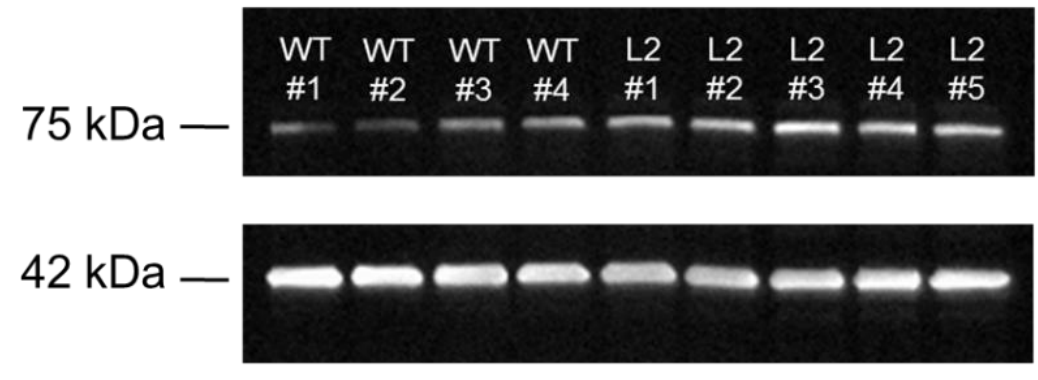

b
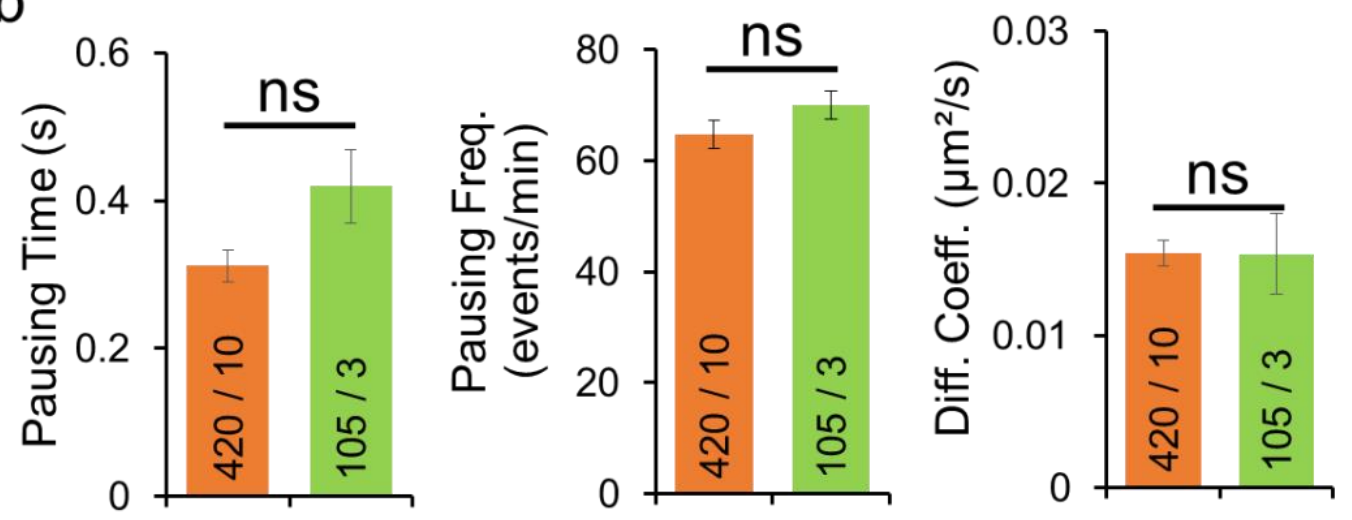

WT

\section{SIc25a12 L2 ${ }^{\text {het }}$}

Supplementary Figure 12 | Quantification of the overexpression of SIc25a12 in the hippocampus in transgenic lines and additional intraneuronal transport parameters.

a, Western blotting was performed on hippocampal tissue dissected from P15 mice from the Slc25a12-transgenic line L2 $2^{\text {het }}$, with comparison to WT mice. WB was carried out with an antibody against Slc25A12 (top) and an antibody against actin (bottom) ( $n=5$ P15 mice both WT and TG). b, Pausing time, pausing frequency and diffusion coefficient displayed no significant differences for the Slc25a12-transgenic lines $\mathrm{L} 2^{\text {het }}(p=0.092, p=0.252$, $p=0.873$, respectively). 